\title{
铜基催化剂电还原 $\mathrm{CO}_{2}$ 为多碳产物的提升策略
}

向开松 ${ }^{1,2}$, 刘雨程 ${ }^{1}$, 于海 ${ }^{2}$, 刘恢 ${ }^{1,3^{*}}$, 李康康 ${ }^{2 *}$

1. 中南大学冶金与环境学院, 长沙 410083;

2. CSIRO Energy, Mayfield West, New South Wales 2304, Australia;

3. 国家重金属污染防治工程技术研究中心, 长沙 410083

*联系人, E-mail: leolau@csu.edu.cn; kangkang.li@csiro.au

2020-01-05 收稿, 2020-02-06 修回, 2020-02-18 接受, 2020-03-06 网络版发表

国家重点研发计划(2017YFC0210405)、湖南创新型省份建设专项(2019RS3006)、CSIRO Julius Career Reward创新项目、中南大学创新驱动 项目(2019CX009)和中南大学研究生自主探索创新项目(2018zzts141)资助

摘要 二氧化碳 $\left(\mathrm{CO}_{2}\right)$ 电催化还原提供了一种使用可再生能源合成燃料和化学原料, 同时控制大气 $\mathrm{CO}_{2}$ 浓度持续上 升的有效方法. 铜 $(\mathrm{Cu})$ 作为少数能有效将 $\mathrm{CO}_{2}$ 电催化还原为能量密度更高、化工价值更大的多碳产物 $\left(\mathrm{C}_{2+}\right)$ 的金属 催化剂, 受到了最广泛的关注. 尽管 $\mathrm{Cu}$ 催化剂在 $\mathrm{CO}_{2}$ 还原领域不断取得重要进展, 但若要突破其在工业领域的应用 瓶颈, 仍需要在 $\mathrm{C}_{2+}$ 产物更高的选择性和电流密度等指标上取得突破. 本文从催化剂本征催化特性、催化环境与电 解装置等方面系统综述了铜基催化剂电还原 $\mathrm{CO}_{2}$ 为多碳产物的提升策略; 总结提出了通过晶面与形貌调控、氧化 态调节、缺陷工程、串联催化及表面修饰等催化剂定向设计以及通过电解质组分、 $\mathrm{pH}$ 和操作压力等优化环境参 数的有效手段提高 $\mathrm{C}_{2+}$ 选择性; 分析了铜基催化剂电还原 $\mathrm{CO}_{2}$ 为多碳产物的提升机制, 前瞻性地介绍了近期电解装 置在 $\mathrm{CO}_{2}$ 电解上的突破, 并对 $\mathrm{Cu}$ 基催化剂电还原 $\mathrm{CO}_{2}$ 未来的发展进行展望.

关键词二氧化碳, 电化学还原, 铜催化剂, 多碳产物 $\left(\mathrm{C}_{2+}\right)$

由二氧化碳 $\left(\mathrm{CO}_{2}\right)$ 引起的温室效应导致了全球气 候异常, 引发了全球前所未有的关注. 虽然太阳能和风 能等可再生能源将为全球提供日益丰富的低碳能源并 减少 $\mathrm{CO}_{2}$ 的排放, 但随着人类活动能源需求的快速增 长, 可再生能源间歇性变化的特性严重制约了其发展. 安全、可靠、稳定的能量存储技术是解决可再生能源 规模化利用的关键. 将间歇性的可再生电力能源或者 过剩的化石电力能源以高密度化学能的形式存储在化 学能源载体中(如乙醇、乙烯等), 不仅可有效解决可再 生能源的能量存储问题, 同时还能减少温室气体 $\mathrm{CO}_{2}$ 排 放、降低对化石燃料的依赖. 鉴于此, $\mathrm{CO}_{2}$ 电化学还原 得到了全世界各国研究者的广泛关注 ${ }^{[1]}$.

近年来, 二氧化碳电还原 $\left(\mathrm{CO}_{2} \mathrm{RR}\right)$ 虽然取得了巨大
进展, 但目前获得的高选择性产物仍以CO和甲酸盐等 $\mathrm{C}_{1}$ 产品为主 ${ }^{[2]}$. 相比于 $\mathrm{C}_{1}$ 产物, 生产长碳链、高能量密 度的碳氢化合物更有利于缓解能源与化工行业对化石 燃料的严重依赖 ${ }^{[3]}$. 在 $\mathrm{CO}_{2} \mathrm{RR}$ 过程中, 铜对 $\mathrm{H}^{*}$ 的吸附能 $\left(\Delta E_{\mathrm{H}^{*}}\right)$ 弱，而对中间产物 $\mathrm{CO}$ 的吸附能 $\left(\Delta E_{\mathrm{CO}^{*}}\right)$ 适中 (图1), 因此 $\mathrm{Cu}$ 可以在一定程度上抑制产氢反应(HER), 强化 $\mathrm{CO}^{*}$ 中间体加氢或 $\mathrm{C}-\mathrm{C}$ 耦合 ${ }^{[4]}$. 基于此特性, $\mathrm{Cu}$ 成 为了极少数可以将 $\mathrm{CO}_{2}$ 转化为多碳 $\left(\mathrm{C}_{2+}\right)$ 烃或醇的金属 催化剂之一.

但目前铜催化剂仍面临着电催化还原 $\mathrm{CO}_{2}$ 的产物 多样、 $\mathrm{C}_{2+}$ 选择性不高等挑战 ${ }^{[5]}$, 因此如何提高多碳产 物的选择性, 特别是高电流密度条件下的选择性, 是当 前铜催化剂研究的趋势与热点. 近期研究表明, C-C的 


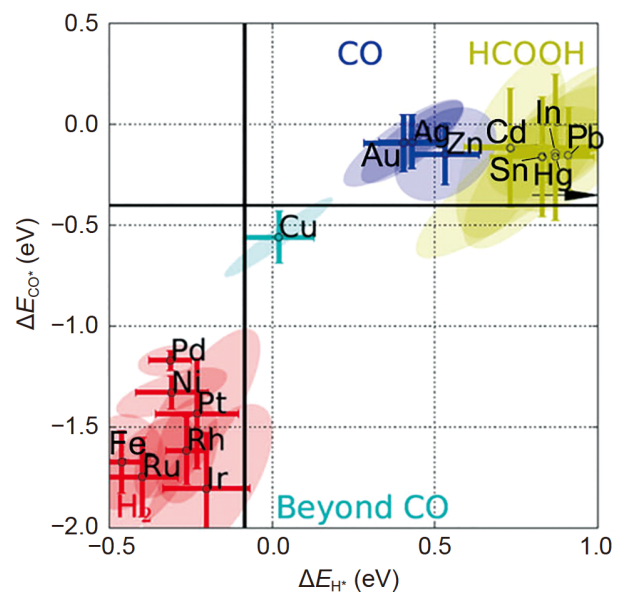

图 1 (网络版彩色)常见金属催化剂上的 $\Delta E_{\mathrm{H}^{*}}$ 与 $\Delta E_{\mathrm{CO}^{*}}{ }^{[4]}$ Figure 1 (Color online) $\Delta E_{\mathrm{H}^{*}}$ and $\Delta E_{\mathrm{CO}^{*}}$ on the common studied metal catalysts $^{[4]}$

耦合是形成 $\mathrm{C}_{2+}$ 的必经过程，而能稳定吸附在铜表面的 $\mathrm{CO}$ *是耦合的关键前驱体之一，其吸附状态对 $\mathrm{C}_{2+}$ 的形 成有直接的影响 ${ }^{[6]}$. 因此, 本文从催化剂设计、电解环 境优化以及电解池装置设计等角度系统论述催化剂表 面 $\mathrm{CO}$ *吸附状态调控方法及 $\mathrm{C}_{2+}$ 产物选择性提升机制, 从而为 $\mathrm{CO}_{2}$ 电催化还原的低成本运行、高价值产出提 供参考途径.

\section{1 催化剂设计}

催化剂是任何催化反应的关键所在. 在电催化还 原 $\mathrm{CO}_{2}$ 过程中, 通过催化剂的设计, 调控关键中间产物 $\mathrm{CO}$ 在催化剂表面的吸附形态，是实现 $\mathrm{CO}_{2}$ 选择性还原 为 $\mathrm{C}_{2+}$ 产物的重要手段. 近年来, 研究者主要从铜催化 剂晶面、形貌、尺寸控制, 氧化态调节, 缺陷工程, 催 化剂串联，表面修饰等诸多方面开展了大量工作并取 得重要进展。

\section{1 晶面、形貌、尺寸控制}

晶面作为一种十分重要的表面结构特征, 对催化有 显著影响 ${ }^{[7]}$. 研究表明, $\mathrm{CO}_{2} \mathrm{RR}$ 的选择性对表面结构极 为敏感: $\mathrm{Cu}(111)$ 晶面上有利于 $\mathrm{CH}_{4}$ 的形成, $\mathrm{Cu}(100)$ 晶面 有利于 $\mathrm{C}_{2} \mathrm{H}_{4}$ 的形成, $\mathrm{Cu}(110)$ 晶面或 $\mathrm{Cu}(751)$ 晶面则有利 于乙醇、醋酸盐等含氧碳氢化合物的生成 ${ }^{[8 \sim 13]}$. 密度泛 函理论(DFT)计算表明, $\mathrm{CO}$ *在 $\mathrm{Cu}(100)$ 晶面更易于吸附, 而且 $C O$ *二聚化形成 $C-C$ 的能垒更低，这是 $\mathrm{Cu}(100)$ 晶 面有利于 $\mathrm{C}_{2} \mathrm{H}_{4}$ 形成的主要原因 ${ }^{[14]}$. 因此, 暴露出更多的 $\mathrm{Cu}(100)$ 晶面成为获取 $\mathrm{C}_{2+}$ 高选择性催化剂的重要途径.
Jiang等人 ${ }^{[15]}$ 通过首先合成易于暴露 $(100)$ 晶面的 $\mathrm{Cu}_{2} \mathrm{O}$ 纳米立方体，再经过还原得到具有优先暴露 $\mathrm{Cu}(100)$ 晶 面的 $\mathrm{Cu}$ 金属催化剂，并获得了 $60 \%$ 以上的 $\mathrm{C}_{2+}$ 法拉第效 率. 另外, $\mathrm{Cu}(511) 、 \mathrm{Cu}(711) 、 \mathrm{Cu}(911) 、 \mathrm{Cu}(810)$ 、 $\mathrm{Cu}(610)$ 等高指数晶面对于 $\mathrm{C}_{2}$ 的选择性均高于 $\mathrm{C}_{1}$ (图2 (a) $)^{[7]}$. 研究指出，高指数晶面含有大量的台阶原子，使 不饱和配位的铜原子比例更高，因此更有利于 $\mathrm{C}_{2+}$ 的形 成. 可以看出, 晶面与产物分布呈现出特定的构效关系. 因此, 晶面的多样性创造了催化位点的多样性, 为 $\mathrm{C}_{1}$ 和 $\mathrm{C}_{2}$ 产物的进一步偶联生成 $\mathrm{C}_{3}$ 提供了结构基础. 如: 相邻 $\mathrm{Cu}(111)$ 和 $\mathrm{Cu}(100)$ 晶面界面可为 $\mathrm{C}_{1}$ 和 $\mathrm{C}_{2}$ 的偶联提供结 合位点, 有利于提高正丙醇 $\left(\mathrm{C}_{3}\right)$ 的法拉第效率 ${ }^{[16]}$.

另外, $\mathrm{CO}_{2} \mathrm{RR}$ 产物的选择性与铜催化剂的尺寸和 形貌有重要关系 ${ }^{[17]}$. 当尺寸小于 $2 \mathrm{~nm}$ 时, $\mathrm{CO}_{2} \mathrm{RR}$ 还原 过程中的产物主要以产 $\mathrm{CO}$ 和 $\mathrm{H}_{2}$ 为主 ${ }^{[18]}$. 当粒径增长到 $5 \sim 15 \mathrm{~nm}$ 时，碳氢化合物开始形成. 而这一现象的出现 源于：当尺寸小于 $2 \mathrm{~nm}$ 时，低配位原子比例急剧上升， 低配位原子与 $\mathrm{CO}^{*} 、 \mathrm{H}^{*}$ 结合过强限制了其后续的加 氢、 $\mathrm{C}-\mathrm{C}$ 耦合等过程. 因此, 若要提高 $\mathrm{CO}_{2} \mathrm{RR}$ 法拉第效 率, 需将铜催化剂纳米尺寸控制在 $2 \mathrm{~nm}$ 以上. 只有当粒 径大于 $10 \mathrm{~nm}$ 时，较小的粒径才有利于 $\mathrm{C}_{2}$ 产物的形成. 例如, Handoko等人 ${ }^{[19]}$ 的研究表明, 铜催化剂粒径从 $41 \mathrm{~nm}$ 减小到 $18 \mathrm{~nm}, \mathrm{C}_{2} \mathrm{H}_{4}$ 选择性从 $10 \%$ 增大到 $43 \%$. 而 且, 较小尺寸的晶粒对 $\mathrm{CO}^{*}$ 的吸附位点更为多样, 因此 更有利于 $\mathrm{C}_{3}$ 产物的形成.

随着纳米技术的进步，晶面和尺寸均可以通过形 貌来实现对其调控. 当合成具有几十至几百纳米、立 方体形貌的单晶铜时，可暴露众多的 $\mathrm{Cu}(100)$ 晶面，从 而提高 $\mathrm{C}_{2} \mathrm{H}_{4}$ 选择性 ${ }^{[16]}$. 当催化剂具有多级形貌(如纳米 孔道、纳米空穴)时，可将 $\mathrm{CO}_{2} \mathrm{RR}$ 还原的中间产物限域 于催化剂的空间内部，从而提高 $\mathrm{C}-\mathrm{C} 、 \mathrm{C}-\mathrm{H}$ 耦合的概 率, 成为提高 $\mathrm{C}_{2+}$ 产物选择性的新方式(图2(b) $)^{[20,21]}$. 另 外，当铜催化剂表面具有很高的粗糙度时有利于 $\mathrm{C}_{2+}$ 产 物的生成，这与高粗糙度时大比表面积下质子的快速 消耗造成催化剂表面高浓度 $\mathrm{OH}^{-}$有关，而 $\mathrm{OH}^{-}$有利于 $\mathrm{C}-\mathrm{C}$ 耦合产生 $\mathrm{C}_{2+}$ 产物 $^{[21,22]}$.

尽管通过控制晶面、尺寸、形貌等可以有效地提 高 $\mathrm{Cu}$ 对 $\mathrm{C}_{2+}$ 产物的催化选择性, 但是在实际的 $\mathrm{CO}_{2} \mathrm{RR}$ 催 化过程中，晶面、尺寸、形貌随电化学过程的进行而 动态变化, 进而导致催化选择性改变甚至失活 ${ }^{[23-25]}$. 因 此，确保晶面和形貌的稳定性是该策略能否在 $\mathrm{CO}_{2} \mathrm{RR}$ 中实际应用的关键. 

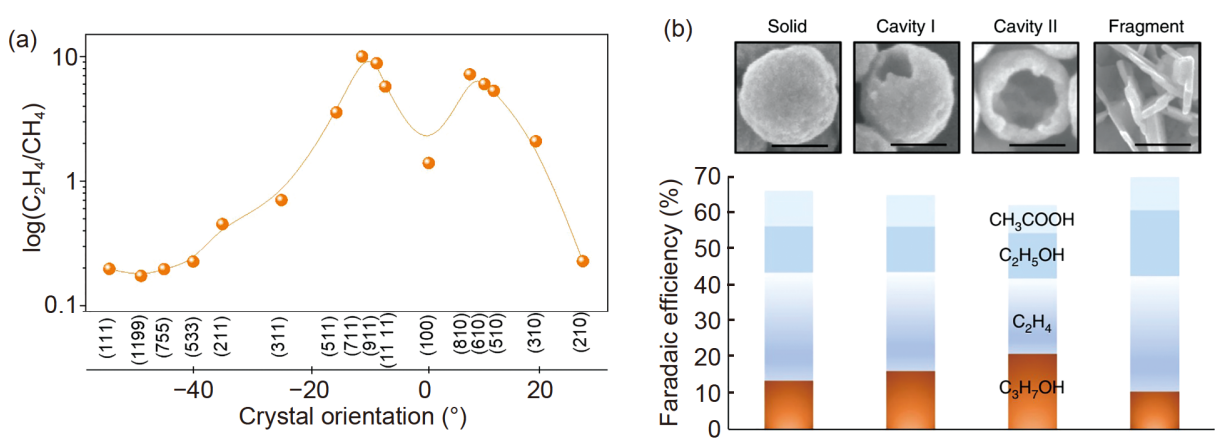

图 2 (网络版彩色)铜晶面与形貌对产物选择性的影响. (a) 不同晶面上 $\mathrm{C}_{2} \mathrm{H}_{4} / \mathrm{CH}_{4}$ 产物比例 ${ }^{[7]}$; (b) 形貌的限域效应提高正丙醇的法拉第效率(标 尺为 $100 \mathrm{~nm})^{[20]}$

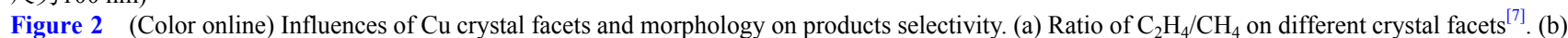
Enhanced n-propanol Faradaic efficiency by confinement effect mediated via morphology (scale bars: $100 \mathrm{~nm})^{[20]}$

\section{2 氧化态调节}

研究发现, 氧化铜经过还原后, 对 $\mathrm{C}_{2+}$ 的选择性有显 著的提高. 部分研究认为, 催化剂性能的变化是因为催 化剂表面存在 $\mathrm{Cu}^{+[26]}$. 同时, 理论计算也表明, 当铜催化 剂表面同时存在 $\mathrm{Cu}^{+}$和 $\mathrm{Cu}^{0}$ 时，两者之间的协同作用将 显著强化 $\mathrm{CO}_{2}$ 活化和 $\mathrm{CO}$ 二聚化的反应过程，从而提高 了 $\mathrm{CO}_{2} \mathrm{RR}$ 的效率和选择性 ${ }^{[27]}$. 但在实际的电解环境中, $\mathrm{Cu}^{+}$易被还原, 催化剂的物相以 $\mathrm{Cu}^{0}$ 为主. 因此, 如何提 高 $\mathrm{Cu}$ 催化剂中 $\mathrm{Cu}^{+}$的含量和其稳定性已经成为许多研 究者提高 $\mathrm{CO}_{2} \mathrm{RR}$ 对 $\mathrm{C}_{2+}$ 选择性的重要策略.
目前主要通过阴离子掺杂和基底效应两种策略来 提高 $\mathrm{Cu}^{+}$含量和稳定性. Sargent课题组 ${ }^{[28]}$ 通过在铜催化 剂中引人嗍(B)元素，并发现B在铜的次表面可以稳定 存在, 因此可诱导 $\mathrm{Cu}^{+}$的稳定存在(图3(a), (b)). B掺杂 的 $\mathrm{Cu}$ 增强了与 $\mathrm{CO}$ *的相互作用, 进一步降低了 $\mathrm{CO}$ *偶 联形成 $\mathrm{O}=\mathrm{C}-\mathrm{C}=\mathrm{O}$ 的能垒，且对于不同晶面的 $\mathrm{Cu}$ 表面 掺杂 $\mathrm{B}$ 都具有相同效应. 在 $\mathrm{CO}_{2} \mathrm{RR}$ 中, $\mathrm{B}$ 掺杂的 $\mathrm{Cu}$ 催化 生成 $\mathrm{C}_{2}$ 产物的法拉第效率可达 $79 \%$, 其 $\mathrm{C}_{2}$ : $\mathrm{C}_{1}$ 产物比值 可达932(图3(c)). 另外, 将金属 $\mathrm{Cu}$ 置于部分基底材料上 时, 铜与基底的界面由于其强相互作用将产生部分 $\mathrm{Cu}^{+}$并稳定存在 ${ }^{[29 ~ 31]}$. 当将铜负载在 $\mathrm{TiO}_{2}$ 载体上时, 它 (a)

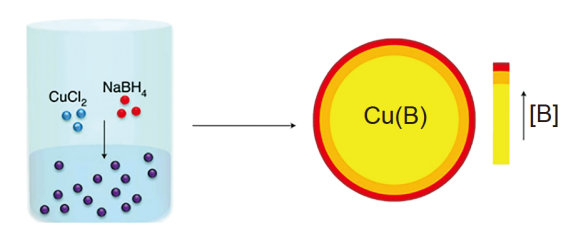

(b)

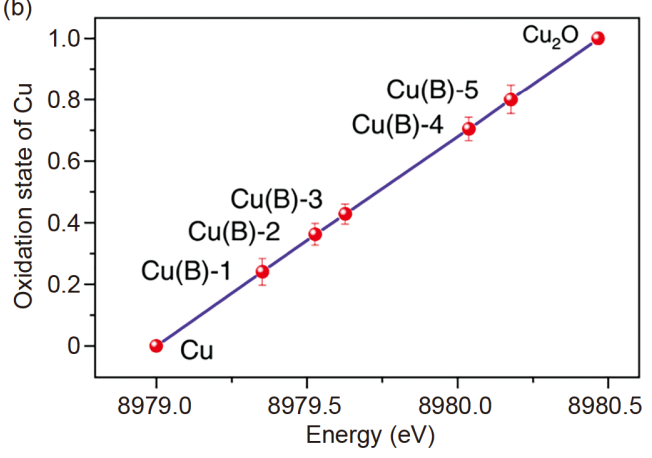

(c)

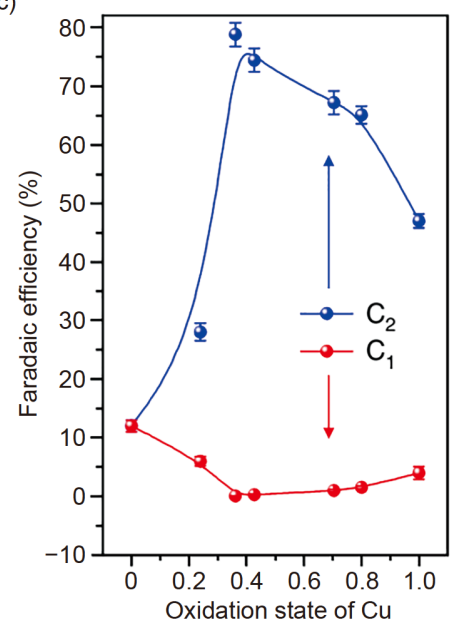

图 3 (网络版彩色)硼掺杂诱导氧化态铜强化 $\mathrm{CO}_{2}$ 还原为 $\mathrm{C}_{2+}{ }^{[28]}$. (a) 硼掺杂铜 $(\mathrm{Cu}(\mathrm{B})$ )的合成示意图; (b) 不同硼掺杂量 $\mathrm{Cu}(\mathrm{B})$ 样品的平均氧化态; (c) 不同氧化态 $\mathrm{Cu}(\mathrm{B})$ 的 $\mathrm{C}_{2}$ 与 $\mathrm{C}_{1}$ 法拉第效率比

Figure 3 (Color online) Boron doping to induce the formation $\mathrm{Cu}$ with oxidation state to enhance the $\mathrm{CO}_{2}$ reduction towards $\mathrm{C}_{2+}{ }^{[28]}$. (a) Schematic diagram of boron doped $\mathrm{Cu}(\mathrm{Cu}(\mathrm{B}))$ preparation. (b) Average oxidation state of $\mathrm{Cu}(\mathrm{B})$ with varied doping contents of boron. (c) Faradaic efficiency ratio of $\mathrm{C}_{2}$ to $\mathrm{C}_{1}$ on $\mathrm{Cu}(\mathrm{B})$ with varied oxidation states 
可以有效地催化 $\mathrm{CO}_{2}$ 还原为含氧碳氢化合物(乙醇、丙 酮和正丙醇), 最大法拉第效率可达 $47.4 \%{ }^{[13]}$.

通过 $\mathrm{Cu}$ 氧化态的调节, 将有效克服晶面、形貌等 不稳定因素带来的催化稳定性不足的问题, 从而表现 出更优异的催化稳定性 ${ }^{[28,32]}$.

\section{3 缺陷工程}

缺陷作为材料中常见的一种晶格失序形式(如晶 界、空位、掺杂等), 在多类反应过程中具有催化效 应 $^{[33]}$. Kanan课题组 ${ }^{[34]}$ 率先发现, 通过电化学还原氧化 铜(oxidize derived $\mathrm{Cu}, \mathrm{OD}-\mathrm{Cu}$ ) 制备的 $\mathrm{Cu}$ 电极在 $\mathrm{CO}_{2}$ 还 原方面的性能明显优于未处理的 $\mathrm{Cu}$ 电极, 由此开启了 缺陷对 $\mathrm{CO}_{2} \mathrm{RR}$ 提升机制的探索和讨论. 后续研究证实 $\mathrm{OD}-\mathrm{Cu}$ 表现出更高的 $\mathrm{C}_{2+}$ 选择性(图4(a)), 但其提升的机 制存在争议 ${ }^{[36]}$. 随着研究的不断深人, Kanan课题 组 ${ }^{[37 \sim 40]}$ 最终确定 $\mathrm{OD}-\mathrm{Cu}$ 中晶界是提升 $\mathrm{CO}_{2}$ 还原性能和 选择性的关键因素. Ager课题组 ${ }^{[35]}$ 的同位素标记实验 结果也从侧面证实, 晶界是催化 $\mathrm{CO}_{2}$ 还原为 $\mathrm{C}_{2} 、 \mathrm{C}_{3}$ 的主 要催化位点. 通过理论计算, 这一现象归因于晶界周围 具有较多的不饱和配位原子, 其可促进 $\mathrm{CO}_{2}$ 的吸附以及 $\mathrm{CO}$ *的二聚化 ${ }^{[41]}$.

作为晶体缺陷的一种, 氧空位不仅有利于在 $\mathrm{CO}_{2}$ 还 原过程中形成* $\mathrm{CO}$ 和* $\mathrm{COH}$ 中间体, 而且有助于* $\mathrm{CH}_{2}$ 的 快速脱附, 加速 $2 * \mathrm{CH}_{2} \rightarrow \mathrm{C}_{2} \mathrm{H}_{4}$ 的转化, 从多种路径强化 $\mathrm{CO}_{2}$ 转化 $\mathrm{C}_{2}$. 富含氧空位的 $\mathrm{CuO}_{x}-\mathrm{Vo}$ 最高可实现 $63 \%$ 的 $\mathrm{C}_{2} \mathrm{H}_{4}$ 法拉第效率 ${ }^{[42]}$.

另外, 异原子的引人也可实现 $\mathrm{C}_{2+}$ 选择性的提升. 外 来原子可改变其周围 $\mathrm{Cu}$ 原子的电子状态, 创造多样的
催化位点. 例如, 金属掺杂可导致 $\mathrm{Cu}$ 产生一定的应变 和配体效应, 使其产生两个相邻但具有不同电子结构 的 $\mathrm{Cu}$ 原子, 分别产生了 $\mathrm{C}_{1}-\mathrm{C}_{1}$ 和 $\mathrm{C}_{1}-\mathrm{C}_{2}$ 耦合的催化位 点, 从而生成更长碳链的产物(图4(b)). 该预测指导下 合成的 $\mathrm{Ag}$ 掺杂的 $\mathrm{Cu}$ 催化剂达到了创纪录的正丙醇法 拉第效率 $(33 \% \pm 1 \%)^{[36]}$.

\section{4 催化剂串联}

由于 $\mathrm{CO}$ 是 $\mathrm{CO}_{2}$ 还原为 $\mathrm{C}_{2+}$ 的关键中间产物, 有效地 串联 $\mathrm{CO}_{2} \rightarrow \mathrm{CO}$ 与 $\mathrm{CO} \rightarrow \mathrm{C}_{2+}$ 两个过程成为近期备受关注 的新策略, 因此, 将产 $\mathrm{CO}$ 的催化剂与能深度还原 $\mathrm{CO}$ 的 $\mathrm{Cu}$ 催化剂组合成为提高 $\mathrm{C}_{2+}$ 选择性的有效手段. 相比于 单一位点催化 $\mathrm{CO}_{2}$ 还原为 $\mathrm{C}_{2+}$, 双位点串联催化的效率 更高. 例如, 在有序分散、无序分散和相分离的双金属 $\mathrm{CuPd}$ 催化剂中, 有序分散型CuPd催化剂通过Pd来调节 临近 $\mathrm{Cu}$ 原子的电子状态, 催化位点弱化了 $\mathrm{Cu}$ 对 $\mathrm{CO}$ 的吸 附, 因此表现出对CO的高选择性. 相分离的 CuPd催化 剂则对CO选择性明显降低, 但 $\mathrm{C}_{2}$ 产物的选择性远超有 序CuPd催化剂(>60\% vs. 3\%, 图5(a)), 这说明CO中间 体在临近 $\mathrm{Cu}$ 原子的表面发生耦合 ${ }^{[43]}$. 鉴于此, 对于这样 的现象建立了一个合理的催化模型, 对于一些双金属 组合的催化剂, $\mathrm{CO}$ 高选择性的催化剂(例如 $\mathrm{Ag}$ 、 $\mathrm{Au}$ 、 $\mathrm{Zn}$ )产生的 $\mathrm{CO}$ 溢流到 $\mathrm{Cu}$ 催化位点上, 可进一步被还原 或偶联(图5(b) $)^{[44-46]}$. 同时, 在 $\mathrm{CO}_{2} \mathrm{RR}$ 过程中, 第二金属 元素产生的 $\mathrm{CO}$ 溢流至 $\mathrm{Cu}$ 表面导致更高的* $\mathrm{CO}$ 覆盖率, 从而降低*CO耦合的能垒, 因此更容易形成 $\mathrm{C}_{2+}$ 产物 $^{[47]}$. 通过该策略, $\mathrm{CuZn} 、 \mathrm{CuAg} 、 \mathrm{CuAu}$ 等催化剂对 $\mathrm{C}_{2+}$ 的选 择性有了显著提升. 不仅如此, 将 $\mathrm{Cu}$ 与产 $\mathrm{CO}$ 的 $\mathrm{N}$ 掺杂的
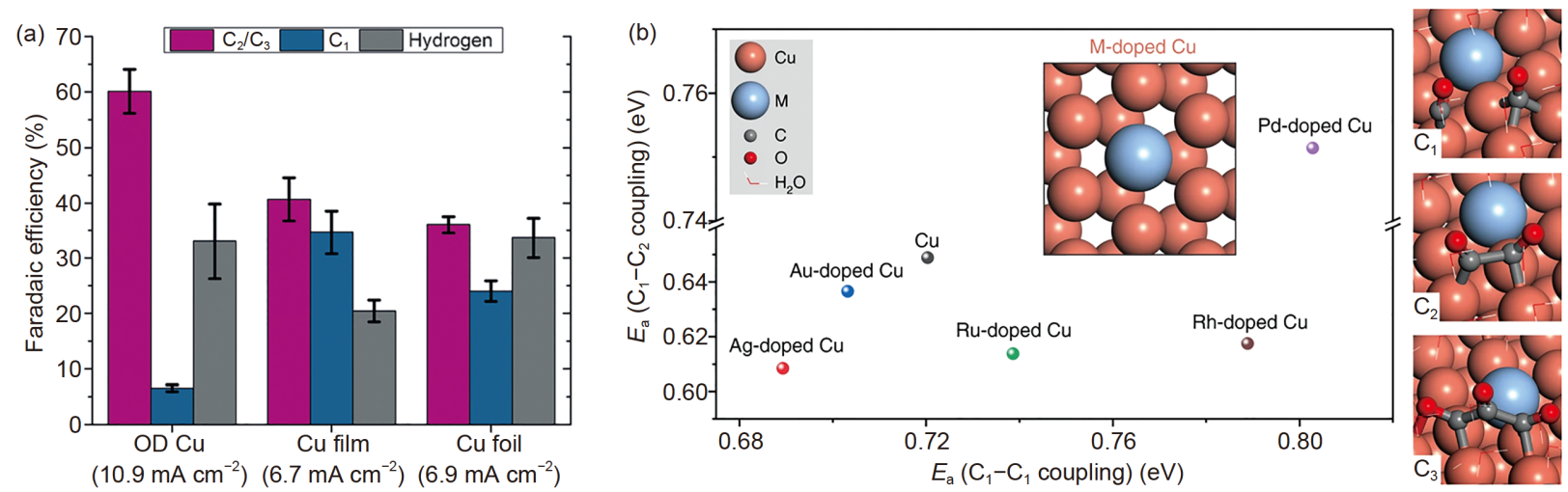

图 4 (网络版彩色) 缺陷设计提高 $\mathrm{C}_{2+}$ 产物选择性 ${ }^{[35]}$. (a) $\mathrm{OD}-\mathrm{Cu}$ 提高铜电极对 $\mathrm{C}_{2}$ 催化选择性 ${ }^{[36]}$; (b) 通过异原子金属掺杂调节 $\mathrm{C}_{1}-\mathrm{C}_{1}$ 与 $\mathrm{C}_{1}-\mathrm{C}_{2}$ 耦 合的的能垒

Figure 4 (Color online) $\mathrm{C}_{2+}$ selectivity improved by defects engineering ${ }^{[35]}$. (a) OD-Cu improved the catalytic selectivity towards $\mathrm{C}_{2+}$ products ${ }^{[36]}$. (b) Regulation of the coupling barrier between $\mathrm{C}_{1}-\mathrm{C}_{1}$ and $\mathrm{C}_{1}-\mathrm{C}_{2}$ by doping foreign metal atoms 

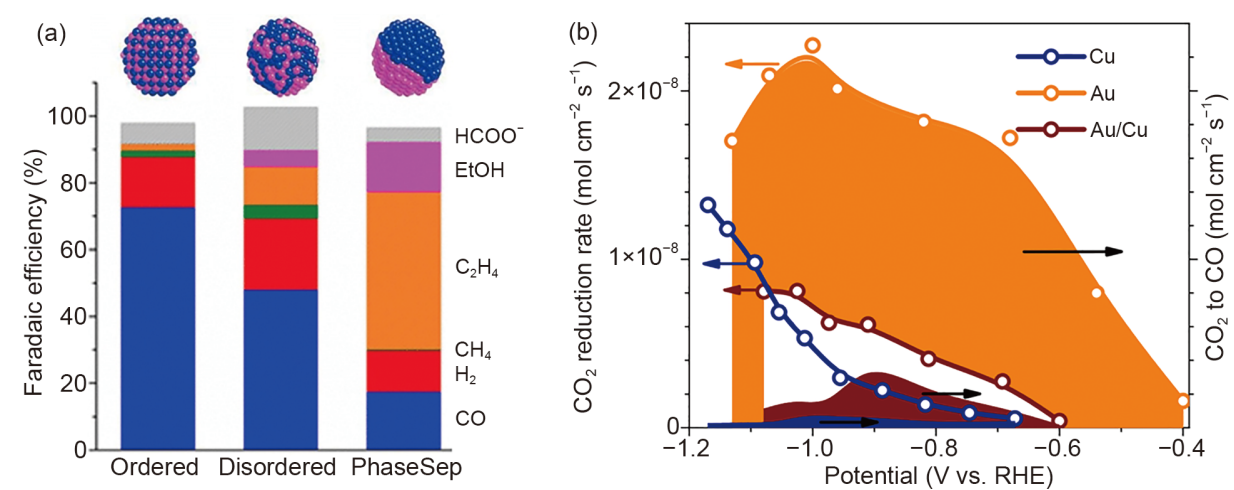

图 5 (网络版彩色)双金属组合强化 $\mathrm{Cu}$ 上 $\mathrm{C}_{2+}$ 的法拉第效率及提升机制. (a) 不同形态 $\mathrm{CuPd}$ 双金属催化剂上产物的法拉第效率 ${ }^{[43]}$; (b) 不同电位 下催化剂上 $\mathrm{CO}_{2}$ 转化与 $\mathrm{CO}$ 消耗速率之间的关系 ${ }^{[44]}$

Figure 5 (Color online) Bi-metal composite improves the $\mathrm{C}_{2+}$ Faradaic efficiency on $\mathrm{Cu}$ and its intensification mechanism. (a) Products Faradaic efficiency on $\mathrm{PdCu}$ bi-metal catalyst with different patterns ${ }^{[43]}$. (b) The relation between $\mathrm{CO}_{2}$ consumption and $\mathrm{CO}$ production rates as a function of the potential $^{[44]}$

碳材料组合时, 通过串联催化机制可以高选择性地获 得乙醇(法拉第效率均在 $60 \%$ 以上 $)^{[48-50]}$, 证明了该策略 的普适性. 若能在催化剂组分的空间分布上进一步优 化, 还可实现更长碳链产物的合成 ${ }^{[51]}$.

\section{5 表面修饰}

除了可以通过掺杂、缺陷等内部改造的方式改变 $\mathrm{CO} *$ 在 $\mathrm{Cu}$ 表面吸附状态外, 铜外部附着的富含电子的 基团也可以实现该目的. 近年来，越来越多的研究发 现 $^{[52 \sim 56]}$, 一些含氮的基团(如咪唑、吡啶以及氨基)可 以有效提高 $\mathrm{Cu}$ 对 $\mathrm{C}_{2+}$ 产物的选择性(图6(a)). 一些理论 计算指出, 这些含氮基团会给铜表面贡献电子, 促进修 饰基团对周围 $\mathrm{CO}$ 的吸附, 激活 $\mathrm{CO}$ 二聚化过程(图 $6(b))^{[56]}$. Han等人 ${ }^{[53]}$ 曾经报道了一种通过添加 $N$-芳基 取代的吡啶鎓化合物来增强电化学还原 $\mathrm{CO}_{2}$ 产 $\mathrm{C}_{2+}$ 的方 法, 在多晶铜电极上沉积上述化合物后, 其对 $\mathrm{C}_{2+}$ 产品
的选择性达到 $70 \% \sim 80 \%$. 近期的研究表明, 可以利用 修饰分子结构差异控制修饰分子的给电子能力来选择 性地调节产物的比例. 修饰分子给电子能力决定了中 间体 $\mathrm{CO}$ 在催化剂上的吸附构型 $\left(\mathrm{CO}_{\text {top }}\right.$ 和 $\left.\mathrm{CO}_{\text {bridge }}\right)$ : 当修 饰分子的给电子较强时会导致 $\mathrm{CO}_{\text {top }}$ 占主导; 反之, 给 电子能力较弱时吸附构型以 $\mathrm{CO}_{\text {bridge }}$ 为主. 由于 $\mathrm{CO}_{\text {top }}$ 倾 向于与 $\mathrm{CO}_{\text {bridge }}$ 偶联, 因此只有当 $\mathrm{CO}_{\text {top }}$ 和 $\mathrm{CO}_{\text {bridge }}$ 比例适 当时才会更容易偶联生成 $\mathrm{C}_{2} \mathrm{H}_{4}{ }^{[52]}$, 合理地解释了修饰 分子与产物选择性之间的关系. 另外, 通过分子修饰提 高铜催化剂表面中间体CO的浓度，也被证实是一种有 效提高 $\mathrm{C}_{2+}$ 产物选择性的途径 ${ }^{[57]}$. 分子修饰可调节催化 剂上中间体的浓度、形态, 为 $\mathrm{Cu}$ 催化剂在 $\mathrm{CO}_{2} \mathrm{RR}$ 过程 中的选择性调控提供了广阔的空间.

\section{2 催化环境优化}

除了催化剂本身对 $\mathrm{CO}$ *有影响之外, 催化剂外围

(b)

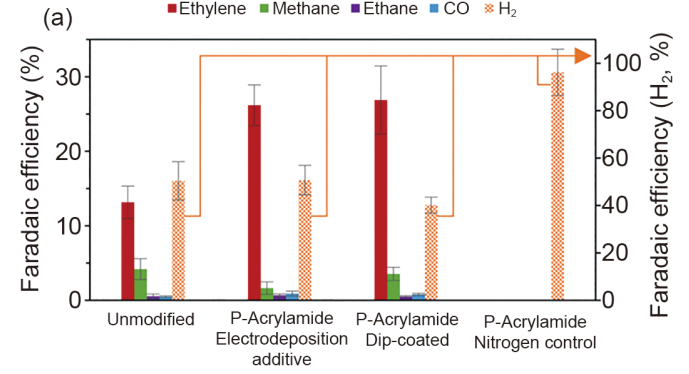

图 6 (网络版彩色)丙烯酰胺的修饰提高Cu对乙烯的法拉第效率及其提升机制 ${ }^{[6]}$. (a) 铜电极修饰丙烯酰胺前后的产物法拉第效率对比; (b) 氨 基促进 $\mathrm{CO}$ 二聚化的机理示意图

Figure 6 (Color online) Improved Faradaic efficiency of ethylene on $\mathrm{Cu}$ via modification of acrylamide and its intensification mechanism ${ }^{[56]}$. (a) Comparison of Faradaic efficiency before and after acrylamide modification. (b) Schematic diagram of the mechanism of amino group promoting CO dimerization 
的电解环境也会影响 $\mathrm{CO}$ *的耦合环境和 $\mathrm{CO} *$ 的覆盖度. $\mathrm{CO}_{2} \mathrm{RR}$ 一般在溶液相中进行, 因此电解质中离子种类 对 $\mathrm{CO}$ 中间体的极化作用有差异而影响了其在催化剂 表面的吸附强度. 溶液中 $\mathrm{pH}$ 环境影响质子在催化剂表 面的吸附与 $\mathrm{CO}$ *覆盖度, 最终影响 $\mathrm{CO}$ *耦合的动力学. 施加的电压决定了不同中间体在催化剂表面的吸附强 度. $\mathrm{CO}_{2}$ 的分压则影响着 $\mathrm{CO}_{2}$ 溶解和传质. 因此, 系统优 化电解的环境参数也是 $\mathrm{CO}_{2} \mathrm{RR}$ 选择性优化的重要方面.

\section{1 电解质的调节作用}

当前主流的电解质以碳酸氢盐，较少考虑电解质 本身对催化的影响. 但阴、阳离子种类会影响催化界 面的局部环境，如 $\mathrm{pH}$ 、极化电场、配位环境等. 研究 显示, 在分别含有 $\mathrm{ClO}_{4}^{-} 、 \mathrm{Cl}^{-} 、 \mathrm{Br}^{-}$与 $\mathrm{I}^{-}$的电解液中, $\mathrm{Cu}$ 对乙烯 $\left(\mathrm{C}_{2} \mathrm{H}_{4}\right)$ 和乙醇 $\left(\mathrm{C}_{2} \mathrm{H}_{5} \mathrm{OH}\right)$ 产物选择性的高低按 照 $\mathrm{ClO}_{4}{ }^{-} \rightarrow \mathrm{Cl}^{-} \rightarrow \mathrm{Br}^{-} \rightarrow \mathrm{I}^{-}$的顺序增加 ${ }^{[58]}$ (图7(a)). 在KI电 解液中, 对于 $\mathrm{C}_{2}$ 和 $\mathrm{C}_{3}$ 产物, 获得了高达 $74 \%$ 的法拉第效 率. 通过原位拉曼发现, 阴离子改变了 $\mathrm{Cu}$ 表面上* $\mathrm{CO}$ 配 位环境 ${ }^{[51]}$. 也有研究表明, 卤素离子可以使铜催化剂表 面 $\mathrm{Cu}^{+}$稳定存在, 因此可以提高 $\mathrm{C}_{2+}$ 的选择性 ${ }^{[59]}$. 另外, 阳离子对 $\mathrm{CO}_{2}$ 还原选择性也有显著影响(图7(b)), 将碳
酸氢盐电解质中的 $\mathrm{Li}^{+}$替换为 $\mathrm{Na}^{+} 、 \mathrm{~K}^{+}$与 $\mathrm{Cs}^{+}$时, $\mathrm{C}_{2} \mathrm{H}_{4}$ 的 选择性由 $21 \%$ 增加至 $25 \% 、 30 \%$ 与 $53 \%(-1.0 \mathrm{~V} \mathrm{vs，}$ RHE). 理论计算揭示, 阳离子半径越大，吸附的阳离子 产生的偶极矩越大, 与 $\mathrm{CO}$ *相互作用最大, 从而增强了 $\mathrm{CO}$ *在 $\mathrm{Cu}$ 表面的吸附 ${ }^{[59]}$. 因此, 阴阳离子的优化成为一 种提高 $\mathrm{C}_{2+}$ 选择性简单有效的手段. 当阴阳离子组合为 $\mathrm{I}^{-}$和 $\mathrm{Cs}^{+}$时, $\mathrm{C}_{2} \mathrm{H}_{4}$ 的法拉第效率可达 $69 \%{ }^{[59]}$. 另外, 在 $\mathrm{Cu}$ 催化剂体系中，低浓度的电解质中具有更高的 $\mathrm{C}_{2}$ 法拉 第效率，其原因更多则归因于低浓度的电解质 $\mathrm{pH}$ 缓冲 能力更低，易于形成催化剂表面局部的高 $\mathrm{pH}$ ，其具体 影响见2.2节。

\section{2 提高催化剂表面pH}

众多的研究表明， $\mathrm{C}_{2+}$ 生成的速控步骤在于 $\mathrm{CO} *$ 的 二聚化, 而 $\mathrm{OH}^{-}$可以促进这一过程的进行 ${ }^{[60,61]}$. 电解过 程中, 由于电解质 $\mathrm{pH}$ 缓冲能力不足造成的高 $\mathrm{pH}$ 促进了 $\mathrm{C}_{2+}$ 产物的形成则间接证实了该理论 ${ }^{[60,62]}$. 在过去的研 究中, 一般是在传统的 $\mathrm{H}$ 型电解池中通过在电解质中溶 解 $\mathrm{CO}_{2}$ 来完成 $\mathrm{CO}_{2} \mathrm{RR}$ 过程. 由于 $\mathrm{CO}_{2}$ 能与 $\mathrm{OH}^{-}$快速反应 生成碳酸根和碳酸氢根, 因此难以直接考察高 $\mathrm{pH}$ 对 $\mathrm{CO}_{2} \mathrm{RR}$ 的影响. 随着电解池的不断改进, 流动式电解池
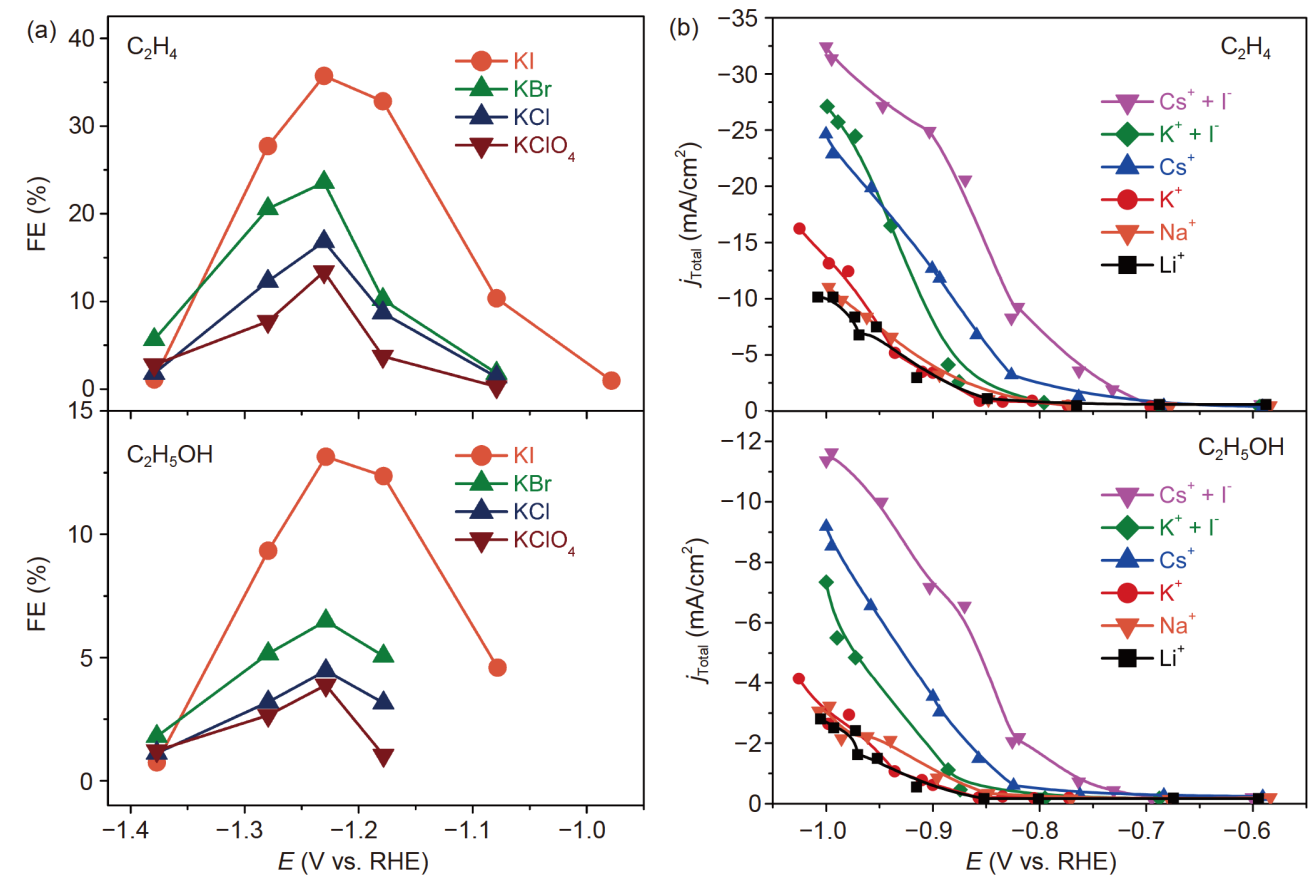

图 7 (网络版彩色)电解质中离子种类对 $\mathrm{C}_{2}$ 法拉第效率的影响. 阴离子 $(\mathrm{a})^{[58]}$ 与阳离子 $(\mathrm{b})^{[59]}$ 对 $\mathrm{C}_{2} \mathrm{H}_{4}$ 和 $\mathrm{C}_{2} \mathrm{H}_{5} \mathrm{OH}$ 催化电流的影响

Figure 7 (Color online) Effect of ions in electrolytes on the $\mathrm{C}_{2+}$ Faradaic efficiency. The effects of anions (a) ${ }^{[58]}$ and cations (b) ${ }^{[59]}$ on the Faradaic efficiency of $\mathrm{C}_{2} \mathrm{H}_{4}$ and $\mathrm{C}_{2} \mathrm{H}_{5} \mathrm{OH}$ 
的出现为研究高 $\mathrm{pH}$ 条件下 $\mathrm{CO}_{2} \mathrm{RR}$ 提供了硬件基础. 流 动式电解池利用气体扩散电极可将气态 $\mathrm{CO}_{2}$ 和电解质 分开并直接在“固体催化剂-气体 $\mathrm{CO}_{2}$-液体电解质”三相 界面上发生反应. Sargent课题组 ${ }^{[63]}$ 使用该构型, 在 $10 \mathrm{~mol} \mathrm{~L}^{-1} \mathrm{KOH}$ 电解质中, 即使在很小的过电位下即 可产生 $\mathrm{C}_{2} \mathrm{H}_{4}$, 在- $0.55 \mathrm{~V}$ (vs. RHE)下可实现高达 $70 \%$ 的 $\mathrm{C}_{2} \mathrm{H}_{4}$ 法拉第效率. 同时, 高浓度的碱性电解质有利于乙 烯生成动力学的进行(图8(a))以及最大程度的抑制析氢 反应(图8(b)), 此性能大幅度提升仅通过电催化剂设计 是难以实现的.

\section{3 电解条件控制}

在 $\mathrm{CO}_{2}$ 转化为 $\mathrm{C}_{2+}$ 产物的过程中, $\mathrm{CO}_{2}$ 还原中间体 $\mathrm{CO}$ *的偶联是其关键的速控步骤, $\mathrm{CO}$ *在催化剂上的 浓度决定了后续转化路径和 $\mathrm{C}_{2+}$ 产物的数量, 而 $\mathrm{CO} *$ 的 生成速率与施加的电压及催化剂表面的 $\mathrm{CO}_{2}$ 浓度有非 常大的关系. 调节电解过程中的电压和通过 $\mathrm{CO}_{2}$ 加压增 加其在催化剂表面的浓度已被证实是提高 $\mathrm{C}_{2}+$ 选择性 的有效手段 ${ }^{[64]}$. $\mathrm{CO}_{2}$ 还原产物均有其特定的还原电位, 因此施加的还原电压直接关系到 $\mathrm{CO}_{2} \mathrm{RR}$ 还原产物的分 布. 不管是 $\mathrm{Cu}_{2} \mathrm{O}$ 衍生的 $\mathrm{Cu}$ 还是单晶 $\mathrm{Cu}$, 其在较低的过 电位即可实现 $\mathrm{CO}$ 的生成, 而 $\mathrm{C}_{2} \mathrm{H}_{4}$ 形成的起始电位比 $\mathrm{CO}_{2} \mathrm{RR}$ 过程中 $\mathrm{CO}$ 生成的起始电位要高300 400 mV. 即 在生成 $\mathrm{C}_{2} \mathrm{H}_{4}$ 之前仅产生了大量的 $\mathrm{CO}$ 气体，而 $\mathrm{CH}_{4}$ 的生 成则处于较高的过电位. $\mathrm{C}_{2} \mathrm{H}_{4}$ 与 $\mathrm{CO}$ (或 $\mathrm{HCOOH}$ )起始电 位的差异和法拉第效率变化规律说明了 $\mathrm{C}_{2} \mathrm{H}_{4}$ 来源于 $\mathrm{CO}$ 进一步还原, 且 $\mathrm{CO}$ *的偶联需要合适的电位范围 (图9(a)), 这也是保证较高 $\mathrm{C}_{2} \mathrm{H}_{4}$ 法拉第效率的前提 ${ }^{[6,65]}$. 另外, Koper课题组 ${ }^{[60]}$ 曾研究过在 $0.5 \mathrm{~mol} \mathrm{~L}^{-1} \mathrm{KHCO}_{3}$ 电解质中不同 $\mathrm{CO}_{2}$ 分压的产物分布, $\mathrm{C}_{2} \mathrm{H}_{4}$ 的法拉效率 从1 atm时的 $11 \%$ 提高至9 atm时的44\%(图9(b)). 尽管较 高的 $\mathrm{CO}_{2}$ 会导致较低的溶液 $\mathrm{pH}$, 但较高的 $\mathrm{CO}_{2}$ 分压增加 了液相中 $\mathrm{CO}_{2}$ 的溶解度, 有利于 $\mathrm{CO}_{2}$ 的电化学还原, 相 应提高了 $\mathrm{CO}$ 在 $\mathrm{Cu}$ 电极上的表面覆盖.

\section{3 电解池结构设计}

传统的 $\mathrm{H}$ 型电解池在评价、篮选催化剂等方面发 挥了重要作用, 但由于 $\mathrm{CO}_{2}$ 其在水溶液体系中有限的物 理溶解度难以在传统的 $\mathrm{H}$ 型电解池中获得较大的催化 电流( $<100 \mathrm{~mA} \mathrm{~cm}^{-2}$ )及较低的析氢效率, 如何解除 $\mathrm{CO}_{2}$ 溶解度的限制是取得突破的关键. 另一方面, 在过去的 大量研究中, $\mathrm{CO}_{2} \mathrm{RR}$ 的研究均以提高产物选择性为主 要目标, 随着产物选择性不断提高, $\mathrm{Cu}$ 在 $\mathrm{C}_{2}$ 的选择性 上已经难以有大的突破. 不但传统的电解池中催化电 流难以达到工业应用的标准 $\left(>200 \mathrm{~mA} \mathrm{~cm}{ }^{-2}\right)$, 而且 $\mathrm{CO}_{2}$ 的利用率以及产物浓度仍处于十分低的水平. 因此, 若 要在大催化电流和较高的 $\mathrm{CO}_{2}$ 单次通过转化率条件下 保证产物选择性, 只能从电解池的结构上进行革新以 满足 $\mathrm{CO}_{2}$ 电还原的规模化应用 ${ }^{[66]}$.

\section{1 流动电解池}

与传统的 $\mathrm{H}$ 型电解池需要溶解 $\mathrm{CO}_{2}$ 不同, 流动电解 池借助了具有疏水功能的气体扩散电极将电解质溶液 和气相 $\mathrm{CO}_{2}$ 分隔到两侧(图10(a)), 无需 $\mathrm{CO}_{2}$ 溶解即可直

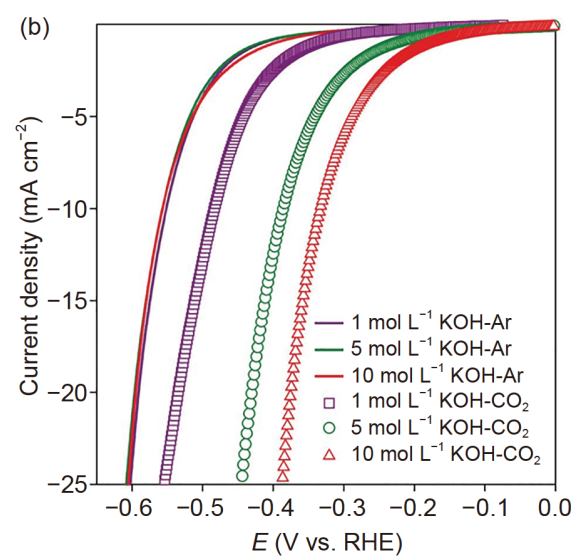

图 8 (网络版彩色) $\mathrm{KOH}$ 浓度对 $\mathrm{CO}_{2}$ 还原的影响 ${ }^{[63]}$. (a) 不同浓度 $\mathrm{KOH}$ 溶液中生成 $\mathrm{C}_{2} \mathrm{H}_{4}$ 的 $\mathrm{Tafel}$ 斜率; (b) 不同浓度 $\mathrm{KOH}$ 溶液中不同气氛下的线性 伏安曲线

Figure 8 (Color online) Effect of $\mathrm{KOH}$ concentration on $\mathrm{CO}_{2}$ reduction ${ }^{[63]}$. (a) Tafel slope of $\mathrm{C}_{2} \mathrm{H}_{4}$ formation with different $\mathrm{KOH}$ concentrations. (b) Linear sweep voltammetry scan in different $\mathrm{KOH}$ electrolyte concentrations in different atmosphere 

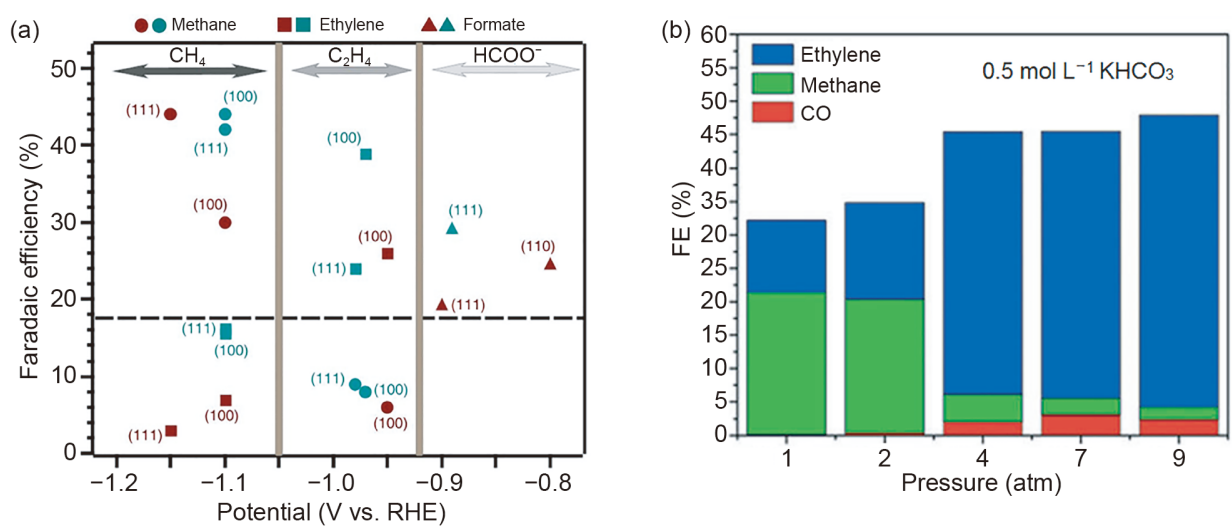

图 9 (网络版彩色)电解条件对产物分布的影响. (a) 不同电位窗口内产物的法拉第效率 ${ }^{[65]}$; (b) $\mathrm{CO}_{2}$ 分压对产物法拉第效率影响 ${ }^{[60]}$

Figure 9 (Color online) Effect of electrolytic conditions on product distribution. (a) Products Faradaic efficiency in different potential windows ${ }^{[65]}$. (b) Effect of $\mathrm{CO}_{2}$ pressure on products Faradaic efficiency ${ }^{[60]}$

(a)
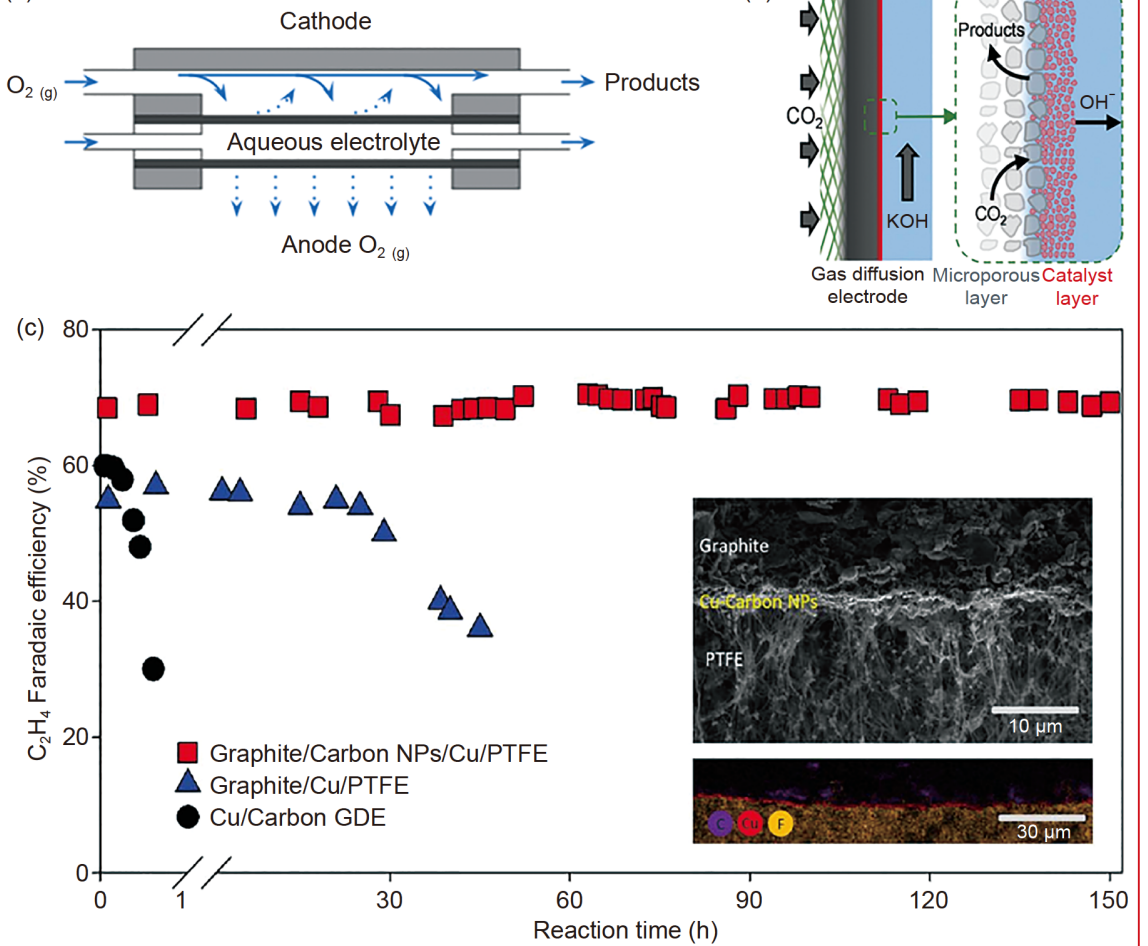

图 10 (网络版彩色)流动电解池的基本构型及其在电解 $\mathrm{CO}_{2}$ 中的应用. (a) 流动电解池的示意图 ${ }^{[67]}$; (b) 流动电解池中气体扩散电极示意图; (c) 气体扩散电极在 $7 \mathrm{~mol} \mathrm{~L}^{-1} \mathrm{KOH}$ 溶液中稳定性测试所得 $\mathrm{C}_{2} \mathrm{H}_{4}$ 法拉第效率. 内部嵌图为气体扩散电极扫描电子显微镜图与对应的元素分布 ${ }^{[63]}$

Figure 10 (Color online) The basic configuration of flow cell and its application in $\mathrm{CO}_{2}$ electrolysis. (a) Schematic diagram of flow cell configuration ${ }^{[67]}$. (b) The schematic illustration of gas diffusion electrode in flow cell. (c) The Faradaic efficiency $\mathrm{C}_{2} \mathrm{H}_{4}$ measured during stability tests with gas diffusion electrodes in $7 \mathrm{~mol} \mathrm{~L}^{-1} \mathrm{KOH}$ solution. The inserted graph shows the scanning electron microscopy (SEM) image of the gas diffusion electrode and the corresponding distribution of elements ${ }^{[63]}$

接以气态形式在催化剂与电解质的相界面处直接转化 (图10(b)). 气液隔离极大提高了 $\mathrm{CO}_{2}$ 在催化剂表面的浓 度，同时可通过提高电解质 $\mathrm{pH}$ 抑制析氢而进一步提高 $\mathrm{C}_{2}+$ 的选择性. Sargent课题组 ${ }^{[63]}$ 通过设计独特结构的 “PTFE/Cu/碳粉/石墨片”气体扩散电极，在 $\mathrm{KOH}$ 电解质 (b)
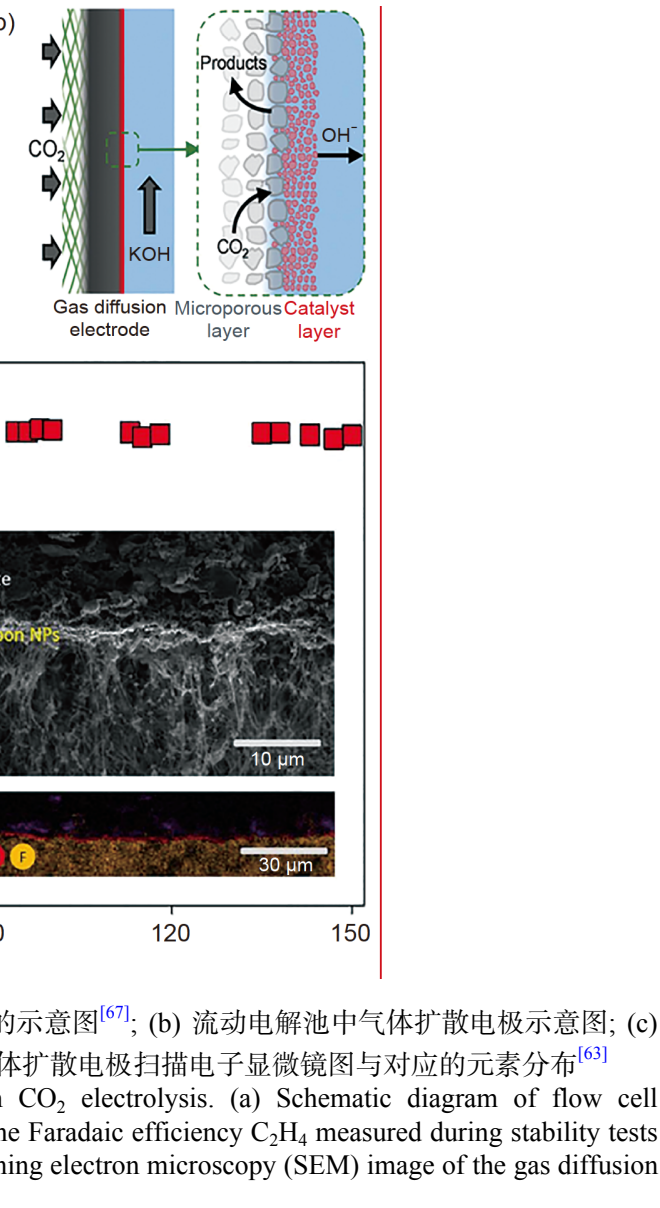
低过电位下工业级别的大催化电流，但依然还面临一 些亟需解决的问题，如气体扩散电极水淹问题会导致 $\mathrm{CO}_{2}$ 的传质再次受阻而降低催化电流 ${ }^{[67]}$, 并加剧析氢 反应. 另外, $\mathrm{CO}_{2}$ 会部分穿透气体扩散电极进入液体电 解质, 降低了 $\mathrm{CO}_{2}$ 的利用率.

\section{2 膜电极电解}

如上述所分析, 流动电解池结构难以避免催化剂 水淹和 $\mathrm{CO}_{2}$ 与液相产物进人液体电解质造成 $\mathrm{CO}_{2}$ 的损 耗和产物分离难题, 如将液体电解质替换为固体电解 质(包括阴离子膜、阳离子膜等), 将有效解决以上问 题. 通过将固体电解质(一般为聚合物电解质)嵌在阴极 和阳极形成三明治结构, 组成膜电极(MEA, 图11(a), (b)). 膜电极可通过膜的组分调节“催化剂-膜”的界面性 质以提高 $\mathrm{C}_{2+}$ 的选择性 ${ }^{[70]}$, 还可物理阻隔原料气体和产 物的穿透, 不但避免了 $\mathrm{CO}_{2}$ 的损耗, 而且利于液相产物

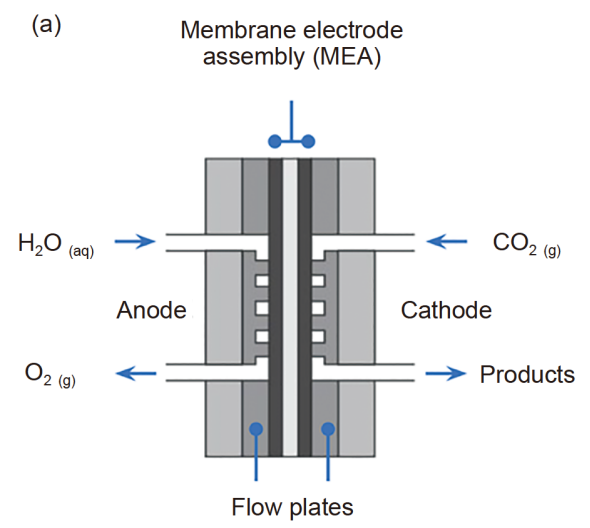

的富集(图11(c)). 因此, 膜电极可直接电解加湿后的 $\mathrm{CO}_{2}$ 气体, 并可通过控制 $\mathrm{CO}_{2}$ 流速、膜组分等产生高浓 度的 $\mathrm{C}_{2+}$ 产物, 减少了后续的产物浓缩分离成本. Sargent课题组 ${ }^{[71]}$ 使用铜催化剂通过碱性膜电极电解实现 了对乙烯和 $\mathrm{C}_{2+}$ 产品 $50 \%$ 和 $80 \%$ 的选择性, 该构型可产 生浓度高达 $30 \%$ 的乙烯和 $4 \mathrm{wt} \%$ 的乙醇产品, 且催化性 能 $\left(>100 \mathrm{~mA} \mathrm{~cm}^{-2}\right.$ ) 可保持 $100 \mathrm{~h}$ 不衰减(图11(d)), $\mathrm{CO}_{2}$ 单 次通过的转化率超过 $30 \%$. Ripatti等人 ${ }^{[72]}$ 也通过膜电极 进行CO电还原, 可实现最高 $68 \%$ 的单次通过转化率, 同 时在 $100 \mathrm{~mA} \mathrm{~cm}^{-2}$ 的电流下可产出 $>1 \mathrm{~mol} \mathrm{~L}^{-1}$ 的醋酸产 品和 $40 \%$ 的乙烯法拉第效率. 随着膜电极在 $\mathrm{CO}_{2}$ 电还原 中的不断应用, 大电流密度下产出高浓度产品将成为 今后 $\mathrm{CO}_{2} \mathrm{RR}$ 还原的趋势.

\section{4 总结与展望}

本文系统综述了近年来以提高 $\mathrm{CO}_{2}$ 电催化还原为

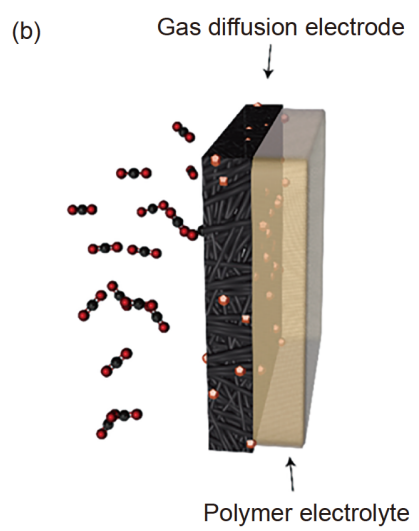

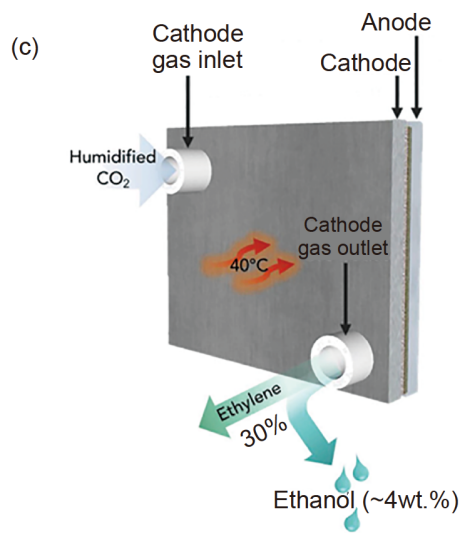

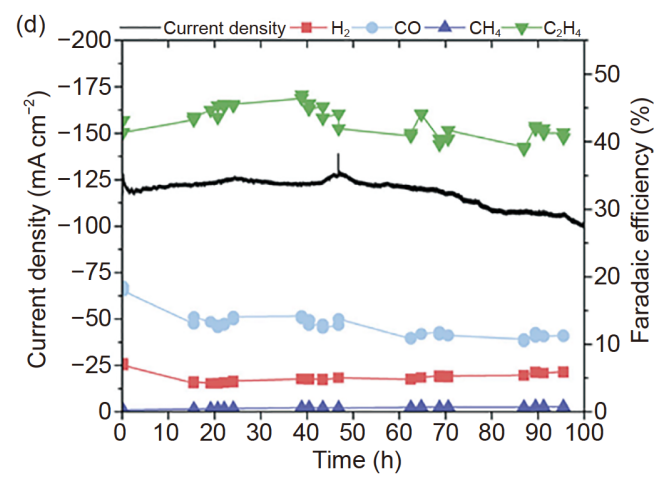

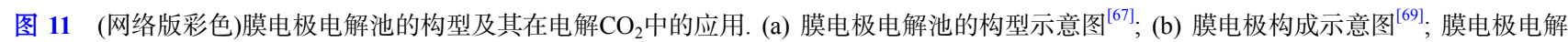
$\mathrm{CO}_{2}$ 生产高浓度 $\mathrm{C}_{2} \mathrm{H}_{4}$ 与 $\mathrm{C}_{2} \mathrm{H}_{5} \mathrm{OH}$ 产品(c) 以及产物的分电流密度与法拉第效率 $(\mathrm{d})^{[71]}$

Figure 11 (Color online) The configuration of membrane electrolyzer and its application in $\mathrm{CO}_{2}$ electrolysis. (a) Schematic configuration of membrane electrolyzer ${ }^{[67]}$. (b) The composing of membrane electrode ${ }^{[69]}$. The membrane electrolyzer applied in $\mathrm{CO}_{2}$ electrolysis to produce concentrated product $\left(\mathrm{C}_{2} \mathrm{H}_{4}, \mathrm{C}_{2} \mathrm{H}_{5} \mathrm{OH}\right)(\mathrm{c})$ and the partial current density and gas products Faradaic efficiency (d) ${ }^{[71]}$ 
多碳产物为研究目标所采用的主要策略, 充分展示了催 化剂设计与改性、电解环境优化以及电解装置构建等 手段在提升多碳产物转化效率方面的优势、机制及拓 展空间, 形成如下结论: (1) 通过物理手段调节催化剂的 形貌、尺寸提高 $\mathrm{CO}$ *吸附位点的比例和多样性; 通过催 化剂的缺陷工程、基团修饰等表面设计强化 $\mathrm{CO}$ *的吸 附; 通过氧化态调节手段改造催化剂内部电子态，优化 $\mathrm{CO}$ 的吸附形态; 通过与产 $\mathrm{CO}$ 材料的复合提高铜表面 $\mathrm{CO}$ *表面富集度，进而提高催化剂对 $\mathrm{C}_{2+}$ 转化的本征活 性. (2) 通过电解质 $\mathrm{pH}$ 、电解质种类的调节降低 $\mathrm{H}^{*}$ 的吸 附、提高 $\mathrm{CO}$ *的吸附强度，通过加压等方式可以提高 $\mathrm{CO}$ *的覆盖度，从而促进 $\mathrm{CO}$ *的耦合，优化上述环境参 数成为提高 $\mathrm{C}_{2+}$ 选择性的重要手段. (3) 通过电解装置的 设计解决了目前传统电解池中 $\mathrm{CO}_{2}$ 溶解度不足的问题, 极大提高了 $\mathrm{CO}_{2}$ 在催化剂表面的传质，扩大了电解质的 选择范围, 可实现大催化电流下 $\mathrm{C}_{2+}$ 产物的高选择性.

目前的研究已经将 $\mathrm{Cu}$ 催化剂的选择性、活性提高 到一个新的高度，但在实用化的道路上依然面临着不 少问题. 如催化剂的规模化制备、催化剂稳定性、产 品的浓度问题等. 另外, 目前关于 $\mathrm{Cu}$ 催化剂上 $\mathrm{CO}_{2}$ 的转 化路径依然存在盲区, 确定 $\mathrm{CO}_{2}$ 向特定产物转化的路径
与其影响因素将为催化剂的设计、电解环境的调整提 供更明确的指导. 基于以上现实问题, 如下几点思路可 为解决上述问题提供部分参考. (1) 膜电极电解池和流 动电解池是今后 $\mathrm{CO}_{2}$ 的电化学还原的理想电解装置, 电 解装置的优化可以减少对铜电极材料纳米尺寸、形 貌、晶面上的依赖，降低铜催化剂的规模化制备成本， 以及降低溶液电解质阻抗造成的能量损耗，同时可以 产出高浓度产物，进一步降低 $\mathrm{CO}_{2} \mathrm{RR}$ 的成本. (2) $\mathrm{CO}_{2}$ 的还原过程中，有少部分 $\mathrm{C}_{2}$ 的生成路径 (如 $2 * \mathrm{CH}_{2} \rightarrow$ $\left.\mathrm{C}_{2} \mathrm{H}_{4}, \quad 2 * \mathrm{CH}_{3} \rightarrow \mathrm{C}_{2} \mathrm{H}_{6}\right)$ 衍生于 $\mathrm{CH}_{4}$ 生成路径 $(* \mathrm{CO} \rightarrow$ ${ }^{*} \mathrm{COH} \rightarrow{ }^{*} \mathrm{C} \rightarrow{ }^{*} \mathrm{CH} \rightarrow{ }^{*} \mathrm{CH}_{2} \rightarrow{ }^{*} \mathrm{CH}_{3} \rightarrow \mathrm{CH}_{4}$ ), 因此可能会 造成催化剂表面的积碳 $\left({ }^{*} \mathrm{C}\right)^{[73]}$. 引导 $\mathrm{CO}_{2}$ 还原通过 $\mathrm{CO}^{*}$ 二聚化路径生成 $\mathrm{C}_{2+}$ 产物, 将避免 $\mathrm{CH}_{4}$ 生成过程中产生 $\mathrm{C}$ 中间体而造成铜催化剂积碳而失活. (3) 机理的研究涉 及催化活性位点和实际中间产物的确定，往往需要一 些原位表征的手段才能实现，如能借助一些现代表征 技术, 如同步辐射X射线光谱、原位红外光谱、原位拉 曼光谱等，将帮助揭示真实催化活性位点和实际中间 产物，确定催化剂参数、电解质组分对中间产物的种 类与后续转化路径的影响，从而指导催化剂的设计和 电解质的选择等.

\section{参考文献}

1 Xu S, Carter E A. Theoretical insights into heterogeneous (photo)electrochemical $\mathrm{CO}_{2}$ reduction. Chem Rev, 2019, 119: 6631-6669

2 Lu Q, Jiao F. Electrochemical $\mathrm{CO}_{2}$ reduction: Electrocatalyst, reaction mechanism, and process engineering. Nano Energy, 2016, 29: 439-456

3 Smith W A, Burdyny T, Vermaas D A, et al. Pathways to industrial-scale fuel out of thin air from $\mathrm{CO}_{2}$ electrolysis. Joule, 2019, 3: 1822-1834

4 Bagger A, Ju W, Varela A S, et al. Electrochemical $\mathrm{CO}_{2}$ reduction: A classification problem. ChemPhysChem, 2017, 18: 3266-3273

5 Shah A H, Wang Y, Hussain $\mathrm{S}$, et al. New aspects of $\mathrm{C}_{2}$ selectivity in electrochemical $\mathrm{CO}_{2}$ reduction over oxide-derived copper. Phys Chem Chem Phys, 2020, 22: 2046-2053

6 Huang Y, Handoko A D, Hirunsit P, et al. Electrochemical reduction of $\mathrm{CO}_{2}$ using copper single-crystal surfaces: Effects of CO* coverage on the selective formation of ethylene. ACS Catal, 2017, 7: 1749-1756

7 Hori Y, Takahashi I, Koga O, et al. Electrochemical reduction of carbon dioxide at various series of copper single crystal electrodes. J Mol Catal AChem, 2003, 199: 39-47

8 Hori Y, Takahashi I, Koga O, et al. Selective formation of $\mathrm{C}_{2}$ compounds from electrochemical reduction of $\mathrm{CO}_{2}$ at a series of copper single crystal electrodes. J Phys Chem B, 2002, 106: 15-17

9 Schouten K J P, Qin Z, Pérez Gallent E, et al. Two pathways for the formation of ethylene in CO reduction on single-crystal copper electrodes. J Am Chem Soc, 2012, 134: 9864-9867

10 Schouten K J P, Kwon Y, van der Ham C J M, et al. A new mechanism for the selectivity to $\mathrm{C}_{1}$ and $\mathrm{C}_{2}$ species in the electrochemical reduction of carbon dioxide on copper electrodes. Chem Sci, 2011, 2: 1902-1909

11 Takahashi I, Koga O, Hoshi N, et al. Electrochemical reduction of $\mathrm{CO}_{2}$ at copper single crystal $\mathrm{Cu}(\mathrm{S})-[n(111) \times(111)]$ and $\mathrm{Cu}(\mathrm{S})-[n(110) \times(100)]$ electrodes. J Electroanal Chem, 2002, 533: 135-143

12 Hahn C, Hatsukade T, Kim Y G, et al. Engineering $\mathrm{Cu}$ surfaces for the electrocatalytic conversion of $\mathrm{CO}_{2}$ : Controlling selectivity toward oxygenates and hydrocarbons. Proc Natl Acad Sci USA, 2017, 114: 5918-5923

13 Yuan J, Zhang J J, Yang M P, et al. $\mathrm{CuO}$ nanoparticles supported on $\mathrm{TiO}_{2}$ with high efficiency for $\mathrm{CO}_{2}$ electrochemical reduction to ethanol. Catalysts, 2018, 8: 171

14 Sandberg R B, Montoya J H, Chan K, et al. CO-CO coupling on Cu facets: Coverage, strain and field effects. Surf Sci, 2016, 654: 56-62 
15 Jiang K, Sandberg R B, Akey A J, et al. Metal ion cycling of Cu foil for selective C-C coupling in electrochemical $\mathrm{CO}_{2}$ reduction. $\mathrm{Nat}_{\mathrm{Catal}}$, 2018, 1: $111-119$

16 Pang Y, Li J, Wang Z, et al. Efficient electrocatalytic conversion of carbon monoxide to propanol using fragmented copper. Nat Catal, 2019, 2: 251-258

17 Tang W, Peterson A A, Varela A S, et al. The importance of surface morphology in controlling the selectivity of polycrystalline copper for $\mathrm{CO}_{2}$ electroreduction. Phys Chem Chem Phys, 2012, 14: 76-81

18 Reske R, Mistry H, Behafarid F, et al. Particle size effects in the catalytic electroreduction of $\mathrm{CO}_{2}$ on Cu nanoparticles. J Am Chem Soc, 2014, 136: 6978-6986

19 Handoko A D, Ong C W, Huang Y, et al. Mechanistic insights into the selective electroreduction of carbon dioxide to ethylene on $\mathrm{Cu}_{2} \mathrm{O}-\mathrm{derived}$ copper catalysts. J Phys Chem C, 2016, 120: 20058-20067

20 Zhuang T T, Pang Y, Liang Z Q, et al. Copper nanocavities confine intermediates for efficient electrosynthesis of $\mathrm{C}_{3}$ alcohol fuels from carbon monoxide. Nat Catal, 2018, 1: 946-951

21 Dutta A, Rahaman M, Luedi N C, et al. Morphology matters: Tuning the product distribution of $\mathrm{CO}_{2}$ electroreduction on oxide-derived Cu foam catalysts. ACS Catal, 2016, 6: 3804-3814

22 Pang Y, Burdyny T, Dinh C T, et al. Joint tuning of nanostructured Cu-oxide morphology and local electrolyte programs high-rate $\mathrm{CO}_{2}$ reduction to $\mathrm{C}_{2} \mathrm{H}_{4}$. Green Chem, 2017, 19: 4023-4030

23 Kim Y G, Baricuatro J H, Javier A, et al. The evolution of the polycrystalline copper surface, first to $\mathrm{Cu}(111)$ and then to $\mathrm{Cu}(100)$, at a fixed $\mathrm{CO}_{2}$ RR potential: A study by Operando EC-STM. Langmuir, 2014, 30: 15053-15056

24 Li Y, Cui F, Ross M B, et al. Structure-sensitive $\mathrm{CO}_{2}$ electroreduction to hydrocarbons on ultrathin 5-fold twinned copper nanowires. Nano Lett, 2017, 17: 1312-1317

25 Liu $\mathrm{H}$, Xiang K, Liu Y, et al. Polydopamine functionalized $\mathrm{Cu}$ nanowires for enhanced $\mathrm{CO}_{2}$ electroreduction towards methane. ChemElectroChem, 2018, 5: 3991-3999

26 Mistry H, Varela A S, Bonifacio C S, et al. Highly selective plasma-activated copper catalysts for carbon dioxide reduction to ethylene. Nat Commun, 2016, 7: 12123

27 Xiao $\mathrm{H}$, Goddard W A, Cheng T, et al. $\mathrm{Cu}$ metal embedded in oxidized matrix catalyst to promote $\mathrm{CO}_{2}$ activation and $\mathrm{CO}$ dimerization for electrochemical reduction of $\mathrm{CO}_{2}$. Proc Natl Acad Sci USA, 2017, 114: 6685-6688

28 Zhou Y, Che F, Liu M, et al. Dopant-induced electron localization drives $\mathrm{CO}_{2}$ reduction to $\mathrm{C}_{2}$ hydrocarbons. Nat Chem, 2018, 10: 974-980

29 Zhang L, Yang S, Lai Y, et al. In-situ synthesis of monodispersed CuO heterostructure on porous carbon monolith for exceptional removal of gaseous $\mathrm{Hg}^{0}$. Appl Catal B-Environ, 2020, 265: 118556

30 Yang S, Liu Z, Yan X, et al. Catalytic oxidation of elemental mercury in coal-combustion flue gas over the $\mathrm{CuAlO}_{2}$ catalyst. Energy Fuels, 2019, 33: 11380-11388

31 Mudiyanselage K, Luo S, Kim H Y, et al. How to stabilize highly active $\mathrm{Cu}^{+}$cations in a mixed-oxide catalyst. Catal Today, 2016, 263: 4-10

32 Yin Z, Yu C, Zhao Z, et al. $\mathrm{Cu}_{3} \mathrm{~N}$ nanocubes for selective electrochemical reduction of $\mathrm{CO}_{2}$ to ethylene. Nano Lett, 2019, 19: 8658-8663

33 Dubau L, Nelayah J, Moldovan S, et al. Defects do catalysis: CO monolayer oxidation and oxygen reduction reaction on hollow PtNi/C nanoparticles. ACS Catal, 2016, 6: 4673-4684

$34 \mathrm{Li} \mathrm{C} \mathrm{W,} \mathrm{Kanan} \mathrm{M} \mathrm{W.} \mathrm{CO}_{2}$ reduction at low overpotential on $\mathrm{Cu}$ electrodes resulting from the reduction of thick $\mathrm{Cu}_{2} \mathrm{O}$ films. J Am Chem Soc, 2012, 134: 7231-7234

35 Wang $\mathrm{X}$, Wang Z, Zhuang T T, et al. Efficient upgrading of $\mathrm{CO}$ to $\mathrm{C}_{3}$ fuel using asymmetric $\mathrm{C}-\mathrm{C}$ coupling active sites. Nat Commun, 2019, 10 : 5186

36 Lum Y, Ager J W. Stability of residual oxides in oxide-derived copper catalysts for electrochemical $\mathrm{CO}_{2}$ reduction investigated with ${ }^{18} \mathrm{O}$ labeling. Angew Chem Int Ed, 2018, 57: 551-554

37 Li C W, Ciston J, Kanan M W. Electroreduction of carbon monoxide to liquid fuel on oxide-derived nanocrystalline copper. Nature, 2014, 508: 504-507

38 Verdaguer-Casadevall A, Li C W, Johansson T P, et al. Probing the active surface sites for CO reduction on oxide-derived copper electrocatalysts. J Am Chem Soc, 2015, 137: 9808-9811

39 Chang C C, Li EY, Tsai M K, et al. A direct grain-boundary-activity correlation for CO electroreduction on Cu nanoparticles. ACS Cent Sci, 2018, 20: $16906-16909$

40 Mariano R G, McKelvey K, White H S, et al. Selective increase in $\mathrm{CO}_{2}$ electroreduction activity at grain-boundary surface terminations. Science, 2017, 358: 1187-1192

41 Garza A J, Bell A T, Head-Gordon M. Is subsurface oxygen necessary for the electrochemical reduction of $\mathrm{CO}_{2}$ on copper? J Phys Chem Lett, 2018, 9: 601-606

42 Gu Z, Yang N, Han P, et al. Oxygen vacancy tuning toward efficient electrocatalytic $\mathrm{CO}_{2}$ reduction to $\mathrm{C}_{2} \mathrm{H}_{4}$. Small Methods, 2019 , 3: 1800449

43 Ma S, Sadakiyo M, Heima M, et al. Electroreduction of carbon dioxide to hydrocarbons using bimetallic $\mathrm{Cu}-\mathrm{Pd}$ catalysts with different mixing patterns. J Am Chem Soc, 2016, 139: 47-50

44 Morales-Guio C G, Cave E R, Nitopi S A, et al. Improved $\mathrm{CO}_{2}$ reduction activity towards $\mathrm{C}_{2+}$ alcohols on a tandem gold on copper electrocatalyst. 
Nat Catal, 2018, 1: 764-771

45 Ren D, Ang B S H, Yeo B S. Tuning the selectivity of carbon dioxide electroreduction toward ethanol on oxide-derived $\mathrm{Cu}_{x} \mathrm{Zn}$ catalysts. ACS Catal, 2016, 6: 8239-8247

46 Gao J, Zhang H, Guo X, et al. Selective C-C coupling in carbon dioxide electroreduction via efficient spillover of intermediates as supported by operando Raman spectroscopy. J Am Chem Soc, 2019, 141: 18704-18714

47 Lum Y, Ager J W. Sequential catalysis controls selectivity in electrochemical $\mathrm{CO}_{2}$ reduction on Cu. Energy Environ Sci, 2018, 11: 2935-2944

48 Song Y, Peng R, Hensley D K, et al. High-selectivity electrochemical conversion of $\mathrm{CO}_{2}$ to ethanol using a copper nanoparticle/N-doped graphene electrode. ChemistrySelect, 2016, 1: 6055-6061

49 Wang $\mathrm{H}$, Tzeng Y K, Ji Y, et al. Synergistic enhancement of electrocatalytic $\mathrm{CO}_{2}$ reduction to $\mathrm{C}_{2}$ oxygenates at nitrogen-doped nanodiamonds/Cu interface. Nat Nanotechnol, 2020, 15: 131-137

50 Han $\mathrm{H}$, Noh $\mathrm{Y}$, Kim $\mathrm{Y}$, et al. Selective electrochemical $\mathrm{CO}_{2}$ conversion to multicarbon alcohols on highly efficient N-doped porous carbonsupported $\mathrm{Cu}$ catalysts. Green Chem, 2020, 22: 71-84

51 O'Mara P B, Wilde P, Benedetti T M, et al. Cascade reactions in nanozymes: Spatially separated active sites inside Ag-core-porous-Cu-shell nanoparticles for multistep carbon dioxide reduction to higher organic molecules. J Am Chem Soc, 2019, 141: 14093-14097

52 Li F, Thevenon A, Rosas-Hernández A, et al. Molecular tuning of $\mathrm{CO}_{2}$-to-ethylene conversion. Nature, 2020, 577: 509-513

53 Han Z, Kortlever R, Chen $\mathrm{H}$ Y, et al. $\mathrm{CO}_{2}$ reduction selective for $\mathrm{C}_{\geqslant 2}$ products on polycrystalline copper with $\mathrm{N}$-substituted pyridinium additives. ACS Cent Sci, 2017, 3: 853-859

54 Xie M S, Xia B Y, Li Y, et al. Amino acid modified copper electrodes for the enhanced selective electroreduction of carbon dioxide towards hydrocarbons. Energy Environ Sci, 2016, 9: 1687-1695

55 Zhong S, Yang X, Cao Z, et al. Efficient electrochemical transformation of $\mathrm{CO}_{2}$ to $\mathrm{C}_{2} / \mathrm{C}_{3}$ chemicals on benzimidazole-functionalized copper surfaces. Chem Commun, 2018, 54: 11324-11327

56 Ahn S, Klyukin K, Wakeham R J, et al. Poly-amide modified copper foam electrodes for enhanced electrochemical reduction of carbon dioxide. ACS Catal, 2018, 8: 4132-4142

57 Li F, Li Y C, Wang Z, et al. Cooperative $\mathrm{CO}_{2}$-to-ethanol conversion via enriched intermediates at molecule-metal catalyst interfaces. Nat Catal, 2020, 3: 75-82

58 Huang Y, Ong C W, Yeo B S. Effects of electrolyte anions on the reduction of carbon dioxide to ethylene and ethanol on copper (100) and (111) surfaces. ChemSusChem, 2018, 11: 3299-3306

59 Gao D, McCrum I T, Deo S, et al. Activity and selectivity control in $\mathrm{CO}_{2}$ electroreduction to multicarbon products over CuO $\mathrm{O}_{x}$ catalysts via electrolyte design. ACS Catal, 2018, 8: 10012-10020

60 Kas R, Kortlever R, Yllmaz H, et al. Manipulating the hydrocarbon selectivity of copper nanoparticles in $\mathrm{CO}_{2}$ electroreduction by process conditions. ChemSusChem, 2015, 2: 354-358

61 Ma S, Sadakiyo M, Luo R, et al. One-step electrosynthesis of ethylene and ethanol from $\mathrm{CO}_{2}$ in an alkaline electrolyzer. J Power Sources, 2016, 301: 219-228

62 Varela A S, Kroschel M, Reier T, et al. Controlling the selectivity of $\mathrm{CO}_{2}$ electroreduction on copper: The effect of the electrolyte concentration and the importance of the local $\mathrm{pH}$. Catal Today, 2016, 260: 8-13

63 Dinh C T, Burdyny T, Kibria M G, et al. $\mathrm{CO}_{2}$ electroreduction to ethylene via hydroxide-mediated copper catalysis at an abrupt interface. Science, 2018, 360: 783-787

64 Hara K. Electrochemical reduction of $\mathrm{CO}_{2}$ on a $\mathrm{Cu}$ electrode under high pressure. J Electrochem Soc, 1994, 141: 2097-2103

65 Ren D, Fong J, Yeo B S. The effects of currents and potentials on the selectivities of copper toward carbon dioxide electroreduction. Nat Commun, 2018, 9: 925

66 Sánchez O G, Birdja Y Y, Bulut M, et al. Recent advances in industrial $\mathrm{CO}_{2}$ electroreduction. Curr Opin Green Sustain Chem, 2019, 16: 47-56

67 Weekes D M, Salvatore D A, Reyes A, et al. Electrolytic $\mathrm{CO}_{2}$ reduction in a flow cell. Acc Chem Res, 2018, 51: 910-918

68 Lv J J, Jouny M, Luc W, et al. A highly porous copper electrocatalyst for carbon dioxide reduction. Adv Mater, 2018, 30: 1803111

69 Higgins D, Hahn C, Xiang C, et al. Gas-diffusion electrodes for carbon dioxide reduction: A new paradigm. ACS Energy Lett, 2019, 4: 317-324

70 Lee J, Lim J, Roh C W, et al. Electrochemical $\mathrm{CO}_{2}$ reduction using alkaline membrane electrode assembly on various metal electrodes. J CO $\mathrm{Util}$, 2019, 31: 244-250

71 Gabardo C M, O'Brien C P, Edwards J P, et al. Continuous carbon dioxide electroreduction to concentrated multi-carbon products using a membrane electrode assembly. Joule, 2019, 3: 2777-2791

72 Ripatti D S, Veltman T R, Kanan M W. Carbon monoxide gas diffusion electrolysis that produces concentrated $\mathrm{C}_{2}$ products with high single-pass conversion. Joule, 2019, 3: 240-256

73 Xiang K, Zhu F, Liu Y, et al. A strategy to eliminate carbon deposition on a copper electrode in order to enhance its stability in $\mathrm{CO}_{2} \mathrm{RR}$ catalysis by introducing crystal defects. Electrochem Commun, 2019, 102: 72-77 


\title{
Strategies to improve the performance of copper-based catalyst for electroreduction of $\mathrm{CO}_{2}$ to multi-carbon products
}

\author{
Kaisong Xiang ${ }^{1,2}$, Yucheng Liu ${ }^{1}$, Hai Yu ${ }^{2}$, Hui Liu ${ }^{1,3^{*}} \&$ Kangkang $\mathrm{Li}^{2^{*}}$ \\ ${ }^{1}$ School of Metallurgy and Environment, Central South University, Changsha 410083, China; \\ ${ }^{2}$ CSIRO Energy, Mayfield West, New South Wales 2304, Australia; \\ ${ }^{3}$ Chinese National Engineering Research Center for Control \& Treatment of Heavy Metal Pollution, Changsha 410083, China \\ * Corresponding authors, E-mail: leolau@csu.edu.cn; kangkang.li@csiro.au
}

Electrocatalytic carbon dioxide $\left(\mathrm{CO}_{2}\right)$ conversion provides an appealing strategy to use renewable electricity to synthesize value-added fuels and chemicals while mitigating $\mathrm{CO}_{2}$ emissions. Copper $(\mathrm{Cu})$ is one of the few metal catalysts that can effectively convert $\mathrm{CO}_{2}$ to high energy density and high-value multi-carbon products $\left(\mathrm{C}_{2^{+}}\right)$, and thus has received increasing attention. Although significant progress has been made in the field of $\mathrm{CO}_{2}$ electroreduction using copper-based catalysts, there are still some bottlenecks when it comes to practical implementation. To enable the commercial application of electrochemical $\mathrm{CO}_{2}$ electroreduction $\left(\mathrm{CO}_{2} \mathrm{RR}\right)$, the high selectivity of $\mathrm{C}_{2+}$ products, low overpotential and high current density are imperatively required. This paper reviews the state-of-the-art research progress of $\mathrm{CO}_{2} \mathrm{RR}$ technology with the attention paid to three important aspects, i.e., catalyst development, catalytic environment optimization and $\mathrm{CO}_{2}$ electrolyzer design, that aim to improve the catalytic performance of electrolytic $\mathrm{CO}_{2}$ conversion.

In the development of efficient and robust catalyst, we identify that the $\mathrm{C}-\mathrm{C}$ coupling is the key step of electrocatalytic $\mathrm{CO}_{2}$ conversion to $\mathrm{C}_{2+}$, which rest with the adsorption state of essential intermediate $\mathrm{CO}^{*}$ on the $\mathrm{Cu}$ catalyst. It is revealed that the selectivity of $\mathrm{C}_{2+}$ is closely related to the adsorption strength of $\mathrm{CO}^{*}$ on catalyst while the adsorption configuration and coverage of $\mathrm{CO}^{*}$ on the $\mathrm{Cu}$ catalyst surface strongly affect the energy barrier for the $\mathrm{CO}^{*}$ dimerization. Based on this understanding, a rational design of catalysts was suggested with the principles of strengthening the adsorption between $\mathrm{CO}^{*}$ and catalyst, adjusting the adsorption configuration of $\mathrm{CO}^{*}$, increasing the coverage of $\mathrm{CO}^{*}$ on $\mathrm{Cu}$ surface, and suppressing the adsorption of $\mathrm{H}^{*}$ on catalyst to minimize hydrogen evolution.

In terms of specific strategy of catalyst development, we recommended the following approaches to regulate the $\mathrm{CO}^{*}$ adsorption on the $\mathrm{Cu}$ surface: (1) Optimizing the $\mathrm{Cu}$ catalyst with crystal facet being $\mathrm{Cu}(100)$ and/or high index facet to match an optimized $\mathrm{CO}^{*}$ absorption configuration for an improved $\mathrm{C}_{2+}$ selectivity; (2) tuning size of catalyst (>10 nm) and the surface roughness/flexural morphology to suppress the $\mathrm{H}^{+}$adsorption and promote the adsorption of $\mathrm{CO}^{*}$ or other $\mathrm{C}_{2+}$ precursors; (3) creating the oxidation state of $\mathrm{Cu}$ by doping or adding a metal oxide support to decrease the $\mathrm{C}-\mathrm{C}$ coupling barrier; (4) increasing the defects on the $\mathrm{Cu}$ surface by destroying the crystal structure or doping effective atoms to create the stronger $\mathrm{CO}^{*}$ adsorption sites and sites' diversity; (5) combining a second catalyst material in tandem with $\mathrm{Cu}$ which facilitates the formation of $\mathrm{CO}$ and subsequent promotion of the kinetics in $\mathrm{C}-\mathrm{C}$ coupling; (6) functionalizing the $\mathrm{Cu}$ surface with electron-rich groups such as amino group to adjust the $\mathrm{CO}^{*}$ adsorption affinity to enhance the $\mathrm{CO}^{*}$ dimerization process.

In parallel with catalyst design, the catalytic environment including electrolyte properties and operation conditions should be optimized with the following approaches suggested to improve the $C_{2+}$ selectivity: (1) Using the supporting electrolyte with larger ion radius of cation or anion to enhance the binding between the $\mathrm{CO}^{*}$ and $\mathrm{Cu}$ surface; (2) increasing the partial pressure of $\mathrm{CO}_{2}$ to increase the aqueous $\mathrm{CO}_{2}$ concentration and subsequently facilitates the $\mathrm{CO}_{2} \mathrm{RR}$ and the yield of $\mathrm{CO}^{*}$ for further reduction; (3) using high-pH electrolyte in the $\mathrm{CO}_{2}$ electrolysis system to promote the $\mathrm{CO}$ dimerization whilst suppressing the hydrogen evolution.

Lastly, we briefly introduced the emerging $\mathrm{CO}_{2}$ electrolyzer design that incorporates the gas diffusion electrode (GDL) to overcome the challenge of $\mathrm{CO}_{2}$ mass transfer faced by the conventional $\mathrm{H}$-type $\mathrm{CO}_{2}$ electrolysis system. Instead of limited $\mathrm{CO}_{2}$ solubility in aqueous electrolyte, the GDL allows direct contact between gaseous $\mathrm{CO}_{2}$ and catalyst, thus greatly improved the mass transfer of $\mathrm{CO}_{2}$ onto the catalyst surface for $\mathrm{CO}_{2} \mathrm{RR}$. This new electrolyzer setup also expand the use of high alkaline electrolyte, that enables the $\mathrm{CO}_{2} \mathrm{RR}$ to be operated at high selectivity of $\mathrm{C}_{2+}$ under high current density.

carbon dioxide, electrochemical reduction, copper-based catalyst, multi-carbon product $\left(\mathrm{C}_{2+}\right)$

doi: 10.1360/TB-2020-0014 